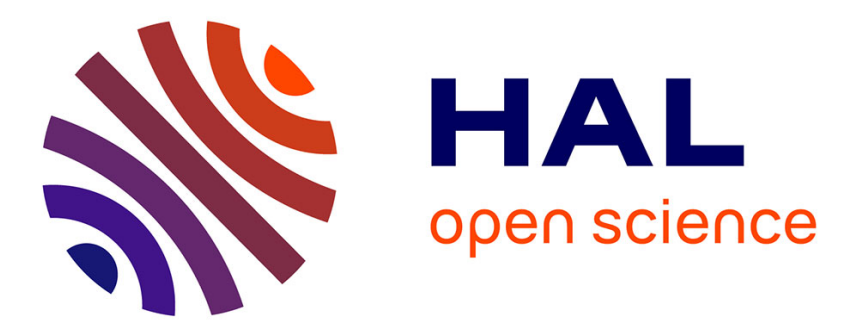

\title{
Speckle observations with PISCO in Merate (Italy): XVI. Astrometric measurements of visual binaries in 2015, and new orbits for DUN 5, ADS 5958, 6276, 7294, 8211, and 13169
}

M. Scardia, Jean-Louis Prieur, L. Pansecchi, R. Argyle, A. Zanutta, E. Aristidi

\section{To cite this version:}

M. Scardia, Jean-Louis Prieur, L. Pansecchi, R. Argyle, A. Zanutta, et al.. Speckle observations with PISCO in Merate (Italy): XVI. Astrometric measurements of visual binaries in 2015, and new orbits for DUN 5, ADS 5958, 6276, 7294, 8211, and 13169. Astronomical Notes / Astronomische Nachrichten, 2018, 339 (7-8), pp.571-585. 10.1002/asna.201813494 . hal-02357166

\section{HAL Id: hal-02357166 https://hal.science/hal-02357166}

Submitted on 9 Nov 2019

HAL is a multi-disciplinary open access archive for the deposit and dissemination of scientific research documents, whether they are published or not. The documents may come from teaching and research institutions in France or abroad, or from public or private research centers.
L'archive ouverte pluridisciplinaire HAL, est destinée au dépôt et à la diffusion de documents scientifiques de niveau recherche, publiés ou non, émanant des établissements d'enseignement et de recherche français ou étrangers, des laboratoires publics ou privés. 


\title{
Speckle observations with PISCO in Merate (Italy): XVI. Astrometric measurements of visual binaries in 2015, and new orbits for DUN 5, ADS 5958, 6276, 7294, 8211 and 13169.
}

\author{
M. Scardia ${ }^{1}$, J.-L. Prieur ${ }^{2,3}$, L. Pansecchi ${ }^{1}$, R.W. Argyle ${ }^{4}$, A. Zanutta ${ }^{1}$, and E. Aristidi ${ }^{5}$ \\ 1 INAF - Osservatorio Astronomico di Brera, Via E. Bianchi 46, 23807 Merate, Italy \\ 2 Université de Toulouse - UPS-OMP - IRAP, Toulouse, France \\ 3 CNRS - IRAP, 14 avenue Edouard Belin, 31400 Toulouse, France \\ 4 Institute of Astronomy, Madingley Road, Cambridge, CB3 0HA, United Kingdom \\ ${ }^{5}$ Université Côte d'Azur, Observatoire de la Côte d'Azur, CNRS, Laboratoire Lagrange, France
}

Received September 16, 2018; accepted

Key words Stars: binaries: close - binaries: visual - astrometry - techniques: interferometric - stars: individual (DUN 5, ADS 4841, ADS 5958, ADS 6276, ADS 7294, ADS 8211, ADS 13169)

\begin{abstract}
We present relative astrometric measurements of visual binaries, made in 2015 with the speckle camera PISCO at the 102-cm Zeiss telescope of Brera Astronomical Observatory, in Merate. Our observing list contains orbital couples as well as binaries whose motion is still uncertain. We obtained 196 new measurements of 173 visual binary stars, with angular separations in the range $0^{\prime \prime} .27-11.3^{\prime \prime}$, and an average accuracy of $0^{\prime \prime} .019$. The mean error on the position angles is $0^{\circ} .6$. Most of the position angles were determined without the usual $180^{\circ}$ ambiguity with the application of triple-correlation techniques and/or by inspection of the long integration files. We present new revised orbits for DUN 5, ADS 5958, 6276, 7294, 8211 and 13169 , partly derived from PISCO observations. The corresponding estimated values for the masses of those systems are compatible with the spectral types. We also computed new rectilinear elements for ADS 4841 for which the physical connection is doubtful from our (and other recent) observations.
\end{abstract}

\section{Introduction}

This paper presents the results of speckle observations of visual binary stars made in Merate (Italy) in 2015 with the Pupil Interferometry Speckle camera and COronagraph (PISCO) on the 102-cm Zeiss telescope of INAF - Osservatorio Astronomico di Brera (OAB, Brera Astronomical Observatory). It is the sixteenth of a series whose purpose is to contribute to the determination of binary orbits (Scardia et al. 2005, 2006, 2007, 2008a, Prieur et al. 2008, Scardia et al. 2009, Prieur et al. 2009, Scardia et al. 2010, Prieur et al. 2010, Scardia et al. 2011, Prieur et al. 2012, Scardia et al. 2013, Prieur et al. 2014, Scardia et al. 2015a, and Prieur et al. 2017, herein: Papers I to XV). The focal instrument PISCO was developed at Observatoire Midi-Pyrénées (France) and first used at Pic du Midi from 1993 to 1998. It was moved to Merate in 2003 and installed on the INAF Zeiss telescope that was dedicated to binary star observations since that epoch. In summer 2015, it was moved to Calern (Côte d'Azur Observatory) in France. It has been operated there since then. This paper presents the last observations made in Merate in the first semester of 2015, before the transfer of PISCO to Calern.
In Sect. 2, we briefly describe our observations. In Sect. 3, we present and discuss the astrometric measurements. We also compare those measurements with the ephemerides computed with the published orbital elements, when available. In Sect. 3.4 we show that the physical connection of the two components of ADS 4841 is doubtful from our (and other recent) observations. and propose new rectilinear elements. In Sect. 4 we present the new revised orbits that we have computed for DUN 5, ADS 5958, 6276, 7294, 8211 and 13169, and discuss the estimated values for the masses of those systems.

\section{Observations}

The observations were carried out with the PISCO speckle camera and the ICCD (Intensified Charge Coupled Device) detector belonging to Nice University (France). This instrumentation is presented in Prieur et al. (1998) and our observing procedure is described in detail in Paper VI.

Our observing list basically includes all the visual binaries for which new measurements are needed to improve their orbits, that are accessible with our instrumentation. It consists of a few thousands objects. A 

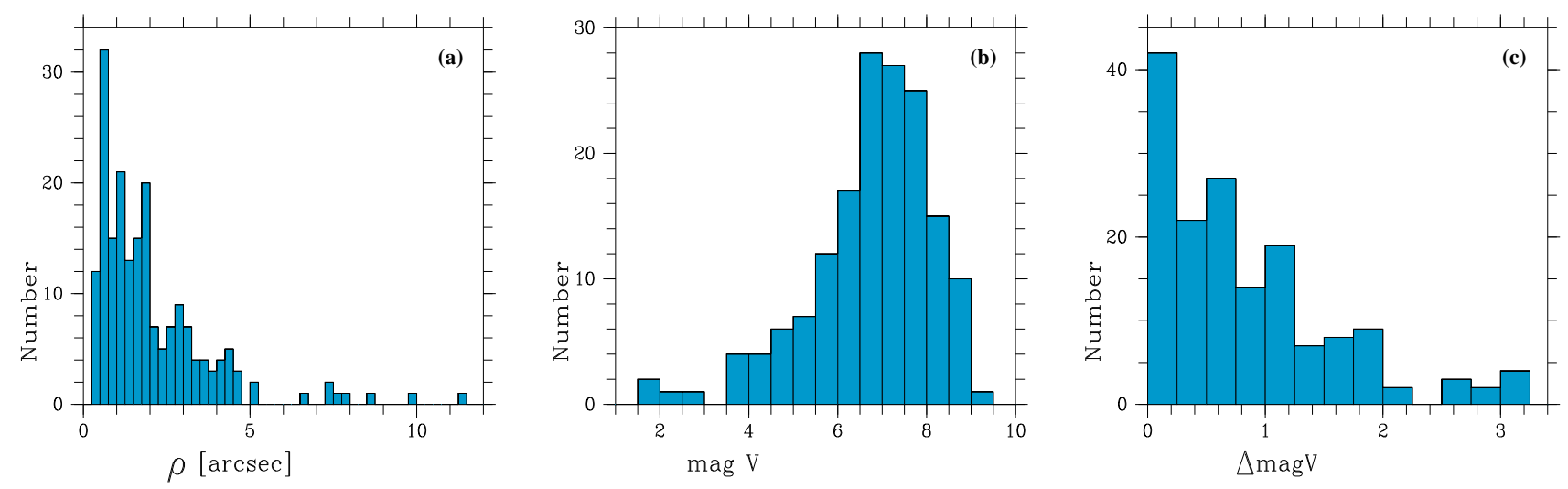

Fig. 1 Distribution of the angular separations of the 196 measurements of Table 1 (a), the total visual magnitudes of the corresponding binaries (b) and the differences of magnitude between their two components (c).

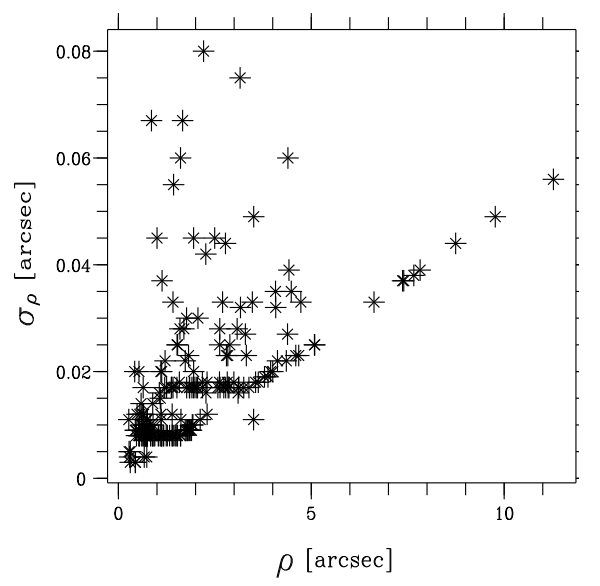

Fig. 2 Error $\sigma_{\rho}$ as a function of the angular separation $\rho$ of the measurements of Table 1 . Our software imposes a lower limitation of the relative errors of $0.5 \%$ to take into account the scale calibration errors.

detailed description can be found in our previous papers (e.g., Paper VI).

The distribution of the angular separations measured in this paper is displayed in Fig. 1a and shows a maximum for $\rho \approx 0^{\prime \prime} .6$. The largest separation of $11^{\prime \prime} .28$ was obtained for SE 5 AC. The smallest separations were measured for ADS 7158 and ADS 5514, with $\rho=0^{\prime \prime} .269$ and $\rho=0^{\prime \prime} .276$, respectively. Let us recall that the diffraction limit is $\rho_{d}=\lambda / D \approx 0^{\prime \prime} .13$ for the Zeiss telescope (aperture $D=1.02 \mathrm{~m}$ ) and the $R$ filter $(\lambda=650 \mathrm{~nm})$.

The distribution of the apparent magnitudes $m_{V}$ and of the difference of magnitudes $\Delta m_{V}$ between the two components are plotted in Figs. 1b and 1c, respectively. The telescope aperture and detector sensitivity led to a limiting magnitude of $m_{V} \approx 9.5$ (Fig. $1 \mathrm{~b}$ ) and

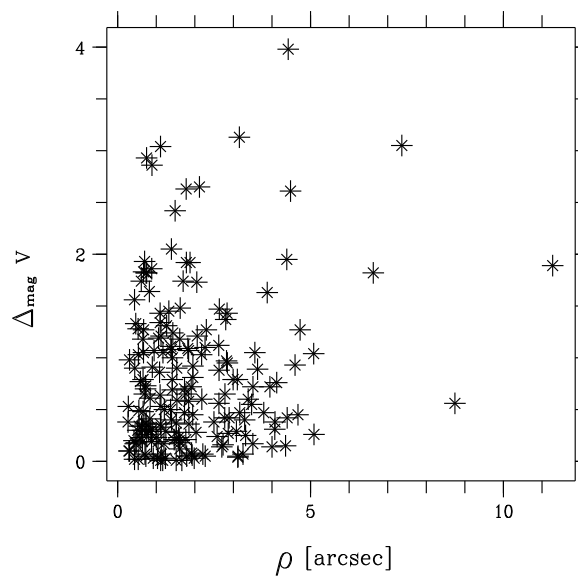

Fig. 3 Difference of magnitude $\Delta_{\text {mag }} \mathrm{V}$ between the two components versus the $\rho$ measurements of Table 1 .

a maximum $\Delta m_{V}$ for speckle measurements of about 3.3 (Fig. 1c).

Using the Hipparcos parallaxes, we were able to construct the HR diagram of those binaries, which is displayed in Fig. 4. We only plotted the 146 objects for which the relative uncertainty on the parallax was smaller than $50 \%$.

\section{Astrometric measurements}

The 196 astrometric measurements obtained with the observations made in 2015 are displayed in Table 1. They concern 173 visual binaries. For each object, we report its WDS name (Washington Double Star Catalog, Mason et al. 2018, hereafter WDS catalog) in Col. 1, the official double star designation in Col. 2 (sequence is "discoverer-number"), and the ADS num- 
Table 1 Table of speckle measurements and O-C residuals with published orbits (begin)

\begin{tabular}{|c|c|c|c|c|c|c|c|c|c|c|c|c|}
\hline WDS & Name & ADS & Epoch & Fil. & $\begin{array}{l}\text { Eyep. } \\
(\mathrm{mm})\end{array}$ & $\begin{array}{c}\rho \\
\left({ }^{\prime \prime}\right)\end{array}$ & $\begin{array}{l}\sigma_{\rho} \\
\left({ }^{\prime \prime}\right)\end{array}$ & $\begin{array}{cc}\theta & \sigma_{\theta} \\
\left(^{\circ}\right) & \left(^{\circ}\right)\end{array}$ & Notes & Orbit & $\begin{array}{r}\Delta \rho(\mathrm{O}-\mathrm{C}) \\
\left({ }^{\prime \prime}\right)\end{array}$ & $\begin{array}{r}\Delta \theta(\mathrm{O}-\mathrm{C}) \\
\left({ }^{\circ}\right)\end{array}$ \\
\hline $00047+3416$ & STF3056AB & 32 & 2015.003 & $\mathrm{R}$ & 20 & 0.711 & 0.008 & $142.6^{*} 0.3$ & & Zir2015a & 0.00 & 0.7 \\
\hline $00063+5826$ & STF3062 & 61 & 2015.003 & $\mathrm{R}$ & 20 & 1.532 & 0.008 & $356.0^{*} 0.3$ & & Sod1999 & -0.02 & -0.0 \\
\hline $00134+2659$ & STT2AB & 161 & 2015.017 & $\mathrm{R}$ & 10 & 0.442 & 0.003 & $157.4^{*} 0.4$ & Elongated & Sca2000b & -0.03 & -2.1 \\
\hline $00209+1059$ & BU1093 & 287 & 2015.017 & $\mathrm{R}$ & 20 & 0.752 & 0.008 & $117.6^{*} 0.3$ & & Lin2010c & -0.01 & -1.6 \\
\hline $00442+4614$ & STF52 & 616 & 2015.017 & $\mathrm{R}$ & 20 & 1.392 & 0.008 & $3.0^{*} 0.3$ & & & & \\
\hline $00491+5749$ & STF60Aa & 671 & 2015.115 & $\mathrm{R}$ & 10 & - & - & - & Unresolv. & & & \\
\hline $01213+1132$ & BU4 & 1097 & 2015.017 & $\mathrm{R}$ & 20 & 0.608 & 0.008 & $106.5^{*} 0.5$ & & Sca2015b & 0.02 & -0.9 \\
\hline$"$ & $"$ & $"$ & 2015.055 & $\mathrm{~W}$ & 20 & 0.612 & 0.008 & $108.2^{*} 1.3$ & & Sca2015b & 0.02 & 0.8 \\
\hline $01532+3719$ & STF179 & 1500 & 2015.017 & $\mathrm{R}$ & 32 & 3.472 & 0.033 & $160.5^{*} 0.3$ & & & & \\
\hline $01559+0151$ & STF186 & 1538 & 2015.017 & $\mathrm{R}$ & 20 & 0.731 & 0.008 & 250.10 .4 & & USN2007b & -0.02 & $-0.1^{Q}$ \\
\hline $02020+0246$ & STF202 & 1615 & 2015.017 & $\mathrm{R}$ & 20 & 1.828 & 0.009 & $263.2^{*} 0.3$ & & Pru2017 & -0.00 & -0.1 \\
\hline$"$ & $"$ & $"$ & 2015.112 & $\mathrm{R}$ & 20 & 1.839 & 0.009 & $263.0^{*} 0.3$ & Elongated & Pru2017 & 0.01 & -0.3 \\
\hline $02037+2556$ & STF208AB & 1631 & 2015.115 & $\mathrm{R}$ & 20 & 1.393 & 0.012 & $343.3^{*} 0.3$ & Diffuse & Hei1996a & -0.10 & -3.4 \\
\hline $02062+2507$ & STF212 & 1654 & 2015.115 & $\mathrm{R}$ & 32 & 1.848 & 0.017 & $161.7^{*} 0.3$ & & & & \\
\hline $02140+4729$ & STF228 & 1709 & 2015.112 & $\mathrm{R}$ & 20 & 0.690 & 0.008 & $299.5^{*} 1.0$ & & Pru2017 & -0.03 & 0.2 \\
\hline $02213+3726$ & STF250 & 1790 & 2015.112 & $\mathrm{R}$ & 32 & 3.164 & 0.032 & $135.6^{*} 0.9$ & & & & \\
\hline $02331+5828$ & STF272 & 1933 & 2015.137 & $\mathrm{R}$ & 32 & 1.984 & 0.017 & $216.0 \quad 0.5$ & & & & \\
\hline $02389+6918$ & STF278 & 1985 & 2015.137 & $\mathrm{R}$ & 20 & 0.520 & 0.009 & $25.9 \quad 1.2$ & & & & \\
\hline $02405+6129$ & STF283AB & 2014 & 2015.137 & $\mathrm{R}$ & 32 & 1.769 & 0.017 & $207.9^{*} 0.4$ & & & & \\
\hline $02422+4242$ & STT44AB & 2052 & 2015.137 & $\mathrm{R}$ & 32 & 1.371 & 0.017 & $57.7^{*} 0.5$ & & & & \\
\hline $02572+0153$ & A2413 & 2236 & 2015.134 & $\mathrm{R}$ & 20 & 0.619 & 0.012 & $163.5^{*} 0.7$ & & Hrt2010a & 0.04 & -0.4 \\
\hline $02589+2137$ & BU525 & 2253 & 2015.134 & $\mathrm{R}$ & 20 & 0.533 & 0.020 & $273.0 \quad 0.6$ & & Rbr2018 & -0.01 & -1.2 \\
\hline $02592+2120$ & STF333AB & 2257 & 2015.113 & $\mathrm{R}$ & 20 & 1.397 & 0.008 & $209.7^{*} 0.3$ & Elongated & FMR2012g & 0.05 & -0.1 \\
\hline $02594+0639$ & STF334 & 2261 & 2015.132 & $\mathrm{R}$ & 20 & 1.123 & 0.008 & $307.5^{*} 0.5$ & & & & \\
\hline $03051+2755$ & STF342 & 2331 & 2015.113 & $\mathrm{R}$ & 32 & 3.291 & 0.027 & $302.1^{*} 0.4$ & & & & \\
\hline $03054+2515$ & STF346AB & 2336 & 2015.115 & $\mathrm{R}$ & 10 & 0.437 & 0.003 & $255.1 \quad 0.3$ & Elongated & Hei1981a & -0.05 & -2.8 \\
\hline $03122+3713$ & STF360 & 2390 & 2015.115 & $\mathrm{R}$ & 32 & 2.866 & 0.017 & $124.2^{*} 0.3$ & & WSI2004a & 0.01 & -1.5 \\
\hline $03127+7133$ & STT50AB & 2377 & 2015.118 & $\mathrm{R}$ & 20 & 1.033 & 0.008 & $147.1^{*} 0.3$ & & Sca2012b & 0.12 & 1.9 \\
\hline $03130+4417$ & STT51 & 2397 & 2015.115 & $\mathrm{R}$ & 20 & 0.601 & 0.010 & $343.6 \quad 1.2$ & & Lin2012a & 0.02 & -2.5 \\
\hline $03140+0044$ & STF367 & 2416 & 2015.118 & $\mathrm{R}$ & 20 & 1.239 & 0.008 & $129.9^{*} 0.3$ & & RAO2015 & 0.00 & -1.0 \\
\hline $03158+5057$ & HU544 & 2425 & 2015.118 & $\mathrm{R}$ & 20 & 1.621 & 0.011 & $100.9^{*} 0.3$ & & & & \\
\hline $03171+4029$ & STF369 & 2443 & 2015.118 & $\mathrm{R}$ & 32 & 3.626 & 0.018 & $29.7^{*} 0.3$ & & & & \\
\hline $03175+6540$ & STT52AB & 2436 & 2015.118 & $\mathrm{R}$ & 20 & 0.555 & 0.012 & $56.5^{*} 1.7$ & & Msn2017e & 0.05 & 2.0 \\
\hline $03177+3838$ & STT53 & 2446 & 2015.118 & $\mathrm{R}$ & 20 & 0.589 & 0.014 & 234.81 .3 & & Msn2017f & -0.01 & -1.0 \\
\hline $03196+6714$ & HU1056 & 2452 & 2015.132 & $\mathrm{R}$ & 32 & 1.200 & 0.017 & $77.7^{*} 1.4$ & & Zir2015a & 0.13 & -2.5 \\
\hline $03206+1911$ & STF377AB & 2478 & 2015.132 & $\mathrm{R}$ & 32 & 1.122 & 0.020 & $108.4^{*} 0.9$ & & & & \\
\hline $03233+2058$ & STF381 & 2504 & 2015.132 & $\mathrm{R}$ & 20 & 1.060 & 0.016 & $108.0^{*} 0.5$ & & & & \\
\hline $03250+4013$ & HU1058 & 2518 & 2015.134 & $\mathrm{R}$ & 20 & 0.843 & 0.009 & $112.5^{*} 0.5$ & & & & \\
\hline $03280+5511$ & STF386 & 2537 & 2015.134 & $\mathrm{R}$ & 32 & 2.700 & 0.033 & $58.5^{*} 0.3$ & & & & \\
\hline $03285+5954$ & STF384AB & 2540 & 2015.132 & $\mathrm{R}$ & 32 & 1.905 & 0.017 & $271.3^{*} 0.6$ & & & & \\
\hline $03287+5026$ & STF388 & 2548 & 2015.134 & $\mathrm{R}$ & 32 & 2.822 & 0.018 & $213.2^{*} 0.3$ & & & & \\
\hline $03293+4503$ & STF391 & 2559 & 2015.118 & $\mathrm{R}$ & 32 & 3.946 & 0.020 & $94.7^{*} 0.3$ & & & & \\
\hline $03302+5922$ & STF389Aa-B & 2563 & 2015.118 & $\mathrm{R}$ & 32 & 2.635 & 0.017 & $70.7^{*} 0.5$ & & & & \\
\hline $03344+2428$ & STF412AB & 2616 & 2015.017 & $\mathrm{R}$ & 20 & 0.744 & 0.008 & $\begin{array}{ll}352.0 & 0.3\end{array}$ & & Sca2002a & -0.01 & 0.3 \\
\hline$"$ & $"$ & $"$ & 2015.091 & $\mathrm{R}$ & 20 & 0.753 & 0.008 & $\begin{array}{lll}351.9 & 0.4\end{array}$ & & Sca2002a & 0.00 & 0.2 \\
\hline$"$ & $"$ & $"$ & 2015.112 & $\mathrm{R}$ & 20 & 0.750 & 0.008 & $351.9 \quad 0.5$ & & Sca2002a & 0.00 & 0.2 \\
\hline $03350+6002$ & STF400AB & 2612 & 2015.017 & $\mathrm{R}$ & 20 & 1.609 & 0.008 & $267.3^{*} 0.4$ & & Msn2017f & 0.02 & -0.6 \\
\hline $03354+3341$ & STF413 & 2625 & 2015.091 & $\mathrm{R}$ & 20 & 1.114 & 0.008 & $123.8^{*} 0.3$ & & & & \\
\hline $03356+3141$ & BU533 & 2628 & 2015.115 & $\mathrm{R}$ & 20 & 1.048 & 0.008 & $220.9^{*} 0.3$ & & Zir2015a & 0.01 & -0.2 \\
\hline $03362+4220$ & A1535 & 2630 & 2015.091 & $\mathrm{R}$ & 20 & 0.723 & 0.008 & $344.9^{*} 0.4$ & & Hrt2008 & 0.00 & 0.4 \\
\hline $03401+3407$ & STF 425 & 2668 & 2015.091 & $\mathrm{R}$ & 20 & 1.915 & 0.010 & $59.5^{*} 0.3$ & Elongated & & & \\
\hline $03407+4601$ & STT59 & 2669 & 2015.115 & $\mathrm{R}$ & 32 & 2.844 & 0.017 & $355.4^{*} 0.5$ & & & & \\
\hline $04159+3142$ & STT77AB & 3082 & 2015.116 & $\mathrm{R}$ & 20 & 0.483 & 0.012 & 298.90 .7 & & Sca1983c & -0.05 & 0.6 \\
\hline
\end{tabular}


Table 1 Table of speckle measurements and O-C residuals with published orbits (cont.)

\begin{tabular}{|c|c|c|c|c|c|c|c|c|c|c|c|c|}
\hline WDS & Name & ADS & Epoch & Fil. & $\begin{array}{l}\text { Eyep. } \\
(\mathrm{mm})\end{array}$ & $\begin{array}{c}\rho \\
\left({ }^{\prime \prime}\right)\end{array}$ & $\begin{array}{l}\sigma_{\rho} \\
\left(^{\prime \prime}\right)\end{array}$ & $\begin{array}{cc}\theta & \sigma_{\theta} \\
\left(^{\circ}\right) & \left(^{\circ}\right)\end{array}$ & Notes & Orbit & $\begin{array}{r}\Delta \rho(\mathrm{O}-\mathrm{C}) \\
\left({ }^{\prime \prime}\right)\end{array}$ & $\begin{array}{r}\Delta \theta(\mathrm{O}-\mathrm{C}) \\
\left({ }^{\circ}\right)\end{array}$ \\
\hline $04179+5847$ & STF511 & 3098 & 2015.116 & $\mathrm{R}$ & 20 & 0.469 & 0.008 & $74.4^{*} 1.1$ & & Hei1996c & -0.04 & -0.2 \\
\hline $04182+2248$ & STF520 & 3114 & 2015.132 & $\mathrm{R}$ & 20 & 0.642 & 0.017 & $80.2 \quad 1.6$ & & Hrt2001b & 0.03 & -2.0 \\
\hline $04218+5002$ & STF521 & 3141 & 2015.132 & $\mathrm{R}$ & 32 & 2.051 & 0.017 & $257.1^{*} 0.4$ & & & & \\
\hline $04225+5136$ & STF522 & 3147 & 2015.137 & $\mathrm{R}$ & 32 & 1.517 & 0.017 & 210.90 .8 & & & & \\
\hline $04233+1123$ & STF535 & 3174 & 2015.135 & $\mathrm{R}$ & 20 & 1.102 & 0.012 & $269.6^{*} 0.3$ & Elongated & Hrt2000c & 0.06 & 2.1 \\
\hline $04239+0928$ & HU304 & 3182 & 2015.113 & $\mathrm{R}$ & 10 & 0.290 & 0.004 & 28.51 .6 & Elongated & Hrt2000b & -0.01 & -1.6 \\
\hline $04268+5539$ & STF531 & 3207 & 2015.135 & $\mathrm{R}$ & 20 & 0.944 & 0.008 & $324.2^{*} 0.5$ & & & & \\
\hline $04301+1538$ & STF554 & 3264 & 2015.113 & $\mathrm{R}$ & 20 & 1.489 & 0.008 & $16.0^{*} 0.6$ & & Baz1980a & -0.10 & 0.8 \\
\hline $04335+1801$ & STF559 & 3297 & 2015.118 & $\mathrm{R}$ & 32 & 3.119 & 0.017 & $275.3^{*} 0.3$ & & & & \\
\hline $04367+1930$ & STF567 & 3330 & 2015.137 & $\mathrm{R}$ & 32 & 2.032 & 0.017 & $344.1^{*} 0.7$ & & USN2002 & 0.00 & 1.1 \\
\hline $04381+4207$ & STF565AB & 3338 & 2015.195 & $\mathrm{R}$ & 20 & 1.326 & 0.008 & $167.4^{*} 0.3$ & & & & \\
\hline $04385+2656$ & STF572AB & 3353 & 2015.195 & $\mathrm{R}$ & 32 & 4.352 & 0.022 & $9.3^{*} 0.3$ & & & & \\
\hline $04400+5328$ & STF566AB-C & 3358 & 2015.137 & $\mathrm{R}$ & 20 & 0.698 & 0.008 & $168.1^{*} 0.3$ & & & & \\
\hline $04422+3731$ & STF577 & 3390 & 2015.137 & $\mathrm{R}$ & 20 & 0.708 & 0.008 & $333.1^{*} 0.4$ & & RAO2015 & 0.01 & 1.4 \\
\hline $04433+5931$ & A1013 & 3391 & 2015.195 & $\mathrm{R}$ & 20 & 0.455 & 0.009 & $294.7 \quad 0.7$ & & Alz1999 & 0.02 & 1.8 \\
\hline $05005+0506$ & STT93 & 3596 & 2015.135 & $\mathrm{R}$ & 32 & 1.535 & 0.025 & $245.9^{*} 0.5$ & & WSI2015 & -0.04 & 2.2 \\
\hline $05079+0830$ & STT98 & 3711 & 2015.137 & $\mathrm{R}$ & 20 & 0.898 & 0.010 & $289.8^{*} 0.3$ & & Sca2008d & -0.04 & -0.9 \\
\hline $05103+3718$ & STF644AB & 3734 & 2015.137 & $\mathrm{R}$ & 20 & 1.614 & 0.008 & $221.6^{*} 0.3$ & & & & \\
\hline $05167+1826$ & STF670A-Bb & 3854 & 2015.132 & $\mathrm{R}$ & 32 & 2.625 & 0.025 & $163.3^{*} 0.3$ & & & & \\
\hline $05172+3320$ & STF666 & 3853 & 2015.132 & $\mathrm{R}$ & 32 & 3.114 & 0.017 & $73.6^{*} 0.3$ & & & & \\
\hline $05247+6323$ & STF677 & 3956 & 2015.116 & $\mathrm{R}$ & 20 & 1.100 & 0.008 & $114.8^{*} 0.3$ & & Hrt2008 & -0.04 & 1.4 \\
\hline $05248+6444$ & STF676 & 3955 & 2015.116 & $\mathrm{R}$ & 32 & 1.415 & 0.017 & $265.1^{*} 0.8$ & & & & \\
\hline $05297+3523$ & HU217 & 4072 & 2015.132 & $\mathrm{R}$ & 20 & 0.652 & 0.012 & $254.3^{*} 2.4$ & & & & \\
\hline $05308+0557$ & STF728 & 4115 & 2015.113 & $\mathrm{R}$ & 20 & 1.269 & 0.008 & $44.7^{*} 0.3$ & & USN1999b & -0.05 & 0.3 \\
\hline $05339+4447$ & STF727 & 4137 & 2015.113 & $\mathrm{R}$ & 32 & 2.176 & 0.018 & $58.4^{*} 0.4$ & & & & \\
\hline $05352+3358$ & AG97 & 4165 & 2015.195 & $\mathrm{R}$ & 32 & 1.733 & 0.022 & $269.9^{*} 0.3$ & & & & \\
\hline $05364+2200$ & STF742 & 4200 & 2015.214 & $\mathrm{R}$ & 32 & 4.078 & 0.032 & $273.2^{*} 0.3$ & & Hop1973b & -0.07 & -2.4 \\
\hline $05371+2655$ & STF749AB & 4208 & 2015.116 & $\mathrm{R}$ & 20 & 1.168 & 0.008 & $\begin{array}{ll}319.6 & 0.3\end{array}$ & & Sca2007a & -0.00 & -0.4 \\
\hline $05371+4150$ & STF736 & 4204 & 2015.214 & $\mathrm{R}$ & 32 & 2.620 & 0.018 & $359.8^{*} 0.4$ & & & & \\
\hline $05399+3757$ & STT112 & 4243 & 2015.211 & $\mathrm{R}$ & 32 & 0.857 & 0.067 & $46.7 \quad 0.5$ & & & & \\
\hline $05535+3720$ & BU1053 & 4472 & 2015.135 & $\mathrm{R}$ & 20 & 1.876 & 0.009 & $359.3^{*} 0.4$ & & Zir2014a & 0.00 & -0.2 \\
\hline $05597+2228$ & STT125 & 4577 & 2015.135 & $\mathrm{R}$ & 20 & 1.421 & 0.008 & $360.0^{*} 0.3$ & & & & \\
\hline $06038+1816$ & HDS824 & - & 2015.135 & $\mathrm{R}$ & 32 & 2.265 & 0.042 & $148.3^{*} 0.3$ & & & & \\
\hline $06149+2230$ & BU1008 & 4841 & 2015.211 & $\mathrm{R}$ & 20 & 1.802 & 0.009 & $256.3^{*} 0.3$ & & Baize (1980) & 0.20 & 3.6 \\
\hline$"$ & $"$ & $"$ & $"$ & $"$ & $"$ & $"$ & $"$ & $" \quad "$ & & This paper & -0.03 & -0.8 \\
\hline$"$ & $"$ & $"$ & 2015.214 & $\mathrm{R}$ & 10 & 1.776 & 0.009 & $256.6^{*} 0.3$ & & Baize (1980) & 0.17 & 4.0 \\
\hline$"$ & $"$ & $"$ & $"$ & $"$ & $"$ & $"$ & $"$ & $" \quad "$ & & This paper & -0.06 & -0.5 \\
\hline $06228+1734$ & STF899 & 4991 & 2015.138 & $\mathrm{R}$ & 32 & 2.181 & 0.017 & $17.8^{*} 0.3$ & & & & \\
\hline $06344+1445$ & STF932 & 5197 & 2015.132 & $\mathrm{R}$ & 32 & 1.580 & 0.018 & $300.8^{*} 0.6$ & & Hop1960a & -0.05 & -0.2 \\
\hline $06364+2717$ & STT149 & 5234 & 2015.269 & $\mathrm{R}$ & 20 & 0.671 & 0.008 & $277.1^{*} 0.4$ & & Hei1993d & -0.07 & -5.4 \\
\hline $06387+4135$ & STF941 & 5269 & 2015.132 & $\mathrm{R}$ & 32 & 1.950 & 0.020 & $82.7^{*} 0.6$ & & & & \\
\hline $06396+2816$ & STT152 & 5289 & 2015.138 & $\mathrm{R}$ & 20 & 0.822 & 0.010 & $35.3^{*} 0.6$ & & & & \\
\hline $06410+0954$ & STF950 & 5322 & 2015.214 & RL & 32 & 3.155 & 0.075 & $213.7^{*} 1.3$ & & & & \\
\hline $06462+5927$ & STF948AB & 5400 & 2015.132 & $\mathrm{R}$ & 20 & 1.895 & 0.009 & $67.0^{*} 0.3$ & & WSI2006b & -0.00 & 0.1 \\
\hline$"$ & STF948AC & 5400 & 2015.132 & $\mathrm{R}$ & 32 & 8.742 & 0.044 & $308.2^{*} 0.3$ & Diffuse & & & \\
\hline$"$ & STF948BC & 5400 & 2015.132 & $\mathrm{R}$ & 32 & 9.769 & 0.049 & $298.6^{*} 0.3$ & & & & \\
\hline $06531+5927$ & STF963 & 5514 & 2015.214 & $\mathrm{RL}$ & 10 & 0.276 & 0.005 & $349.8 \quad 0.6$ & & Sca2008d & -0.00 & 1.0 \\
\hline $06546+1311$ & STF982AB & 5559 & 2015.271 & $\mathrm{R}$ & 32 & 7.366 & 0.037 & $142.8^{*} 0.3$ & & Msn2014b & 0.06 & -0.2 \\
\hline $06573+5825$ & STT159 & 5586 & 2015.214 & $\mathrm{R}$ & 10 & 0.670 & 0.004 & $233.6^{*} 0.3$ & Elongated & Sod1999 & 0.02 & 1.5 \\
\hline $07128+2713$ & STF1037 & 5871 & 2015.116 & $\mathrm{R}$ & 20 & 0.923 & 0.008 & $305.2 \quad 0.3$ & & Sca2015b & -0.02 & -0.8 \\
\hline $07303+4959$ & STF1093 & 6117 & 2015.272 & $\mathrm{R}$ & 20 & 0.878 & 0.008 & $204.7 \quad 0.4$ & & Hrt2009 & -0.01 & 0.3 \\
\hline $07346+3153$ & STF1110 & 6175 & 2015.116 & $\mathrm{R}$ & 32 & 5.081 & 0.025 & $54.4^{*} 0.3$ & & DRs2012 & 0.05 & -0.3 \\
\hline $07401+0514$ & STF1126 & 6263 & 2015.269 & $\mathrm{R}$ & 20 & 0.833 & 0.008 & $175.7^{*} 1.0$ & & Zir2015a & -0.01 & -0.8 \\
\hline $07417+3726$ & STT177 & 6276 & 2015.272 & $\mathrm{R}$ & 20 & 0.530 & 0.008 & $149.3 \quad 1.3$ & & This paper & -0.03 & 2.5 \\
\hline $07573+0108$ & STT185 & 6483 & 2015.288 & $\mathrm{R}$ & 20 & 0.422 & 0.020 & 17.75 .8 & & Msn2009 & 0.03 & -1.6 \\
\hline $08024+0409$ & STF1175 & 6532 & 2015.285 & $\mathrm{R}$ & 32 & 1.430 & 0.055 & $284.7^{*} 0.6$ & & Ole2001 & 0.04 & -6.1 \\
\hline
\end{tabular}


Table 1 Table of speckle measurements and O-C residuals with published orbits (cont.)

\begin{tabular}{|c|c|c|c|c|c|c|c|c|c|c|c|c|c|}
\hline WDS & Name & ADS & Epoch & Fil. & $\begin{array}{l}\text { Eyep. } \\
(\mathrm{mm})\end{array}$ & $\begin{array}{c}\rho \\
\left({ }^{\prime \prime}\right)\end{array}$ & $\begin{array}{l}\sigma_{\rho} \\
\left(^{\prime \prime}\right)\end{array}$ & $\begin{array}{c}\theta \\
\left(^{\circ}\right)\end{array}$ & $\begin{array}{l}\sigma_{\theta} \\
\left(^{\circ}\right)\end{array}$ & Notes & Orbit & $\begin{array}{r}\Delta \rho(\mathrm{O}-\mathrm{C}) \\
\quad\left({ }^{\prime \prime}\right)\end{array}$ & $\begin{array}{r}\Delta \theta(\mathrm{O}-\mathrm{C}) \\
\left({ }^{\circ}\right)\end{array}$ \\
\hline $08041+3302$ & STT187 & 6549 & 2015.285 & $\mathrm{R}$ & 20 & 0.437 & 0.009 & 337.9 & 2.1 & & Msn1999a & 0.02 & 0.4 \\
\hline $08056+2732$ & STF1177 & 6569 & 2015.285 & $\mathrm{R}$ & 32 & 3.507 & 0.049 & $349.7^{*}$ & * 0.3 & & & & \\
\hline $08061-0047$ & A1971 & 6582 & 2015.302 & $\mathrm{R}$ & 32 & 1.001 & 0.045 & $2.7^{*}$ & * 1.0 & & Tok2015c & 0.04 & 0.3 \\
\hline $08095+3213$ & STF1187 & 6623 & 2015.302 & $\mathrm{R}$ & 20 & 3.102 & 0.016 & $21.0^{*}$ & * 0.3 & & Ole2001 & 0.15 & 0.6 \\
\hline $08213-0136$ & STF1216 & 6762 & 2015.274 & $\mathrm{R}$ & 20 & 0.535 & 0.009 & 305.0 & 0.7 & & Tok2014a & 0.01 & -3.2 \\
\hline$"$ & $"$ & $"$ & 2015.280 & $\mathrm{R}$ & 20 & 0.537 & 0.008 & $306.0^{*}$ & * 0.6 & & Tok2014a & 0.02 & -2.2 \\
\hline $08432+3849$ & BU209 & 6946 & 2015.307 & $\mathrm{R}$ & 32 & 1.249 & 0.017 & $9.9^{*}$ & * 1.6 & & & & \\
\hline $08508+3504$ & STF1282 & 7034 & 2015.307 & $\mathrm{R}$ & 32 & 3.504 & 0.011 & $277.5^{*}$ & * 0.9 & & & & \\
\hline $08531+5457$ & A1584 & 7054 & 2015.280 & $\mathrm{R}$ & 20 & 0.673 & 0.008 & $88.9^{*}$ & * 1.2 & & Msn2014a & -0.00 & -3.0 \\
\hline $08542+3035$ & STF1291 & 7071 & 2015.280 & $\mathrm{R}$ & 20 & 1.517 & 0.008 & $309.3^{*}$ & * 0.4 & & & & \\
\hline $08554+7048$ & STF1280 & 7067 & 2015.280 & $\mathrm{R}$ & 32 & 3.078 & 0.028 & $355.4^{*}$ & * 0.3 & & Hei1997 & 0.06 & -0.9 \\
\hline $09012+0245$ & STF1302AB & 7141 & 2015.280 & $\mathrm{R}$ & 32 & 2.732 & 0.017 & $235.7^{*}$ & * 0.3 & & & & \\
\hline $09013+1516$ & STF1300 & 7139 & 2015.288 & $\mathrm{R}$ & 32 & 5.089 & 0.025 & $179.1^{*}$ & * 0.3 & & Zir2008 & 0.05 & 0.0 \\
\hline $09014+3215$ & STF1298 & 7137 & 2015.304 & $\mathrm{R}$ & 32 & 4.481 & 0.035 & $134.9^{*}$ & * 0.4 & & & & \\
\hline $09020+0240$ & BU211 & 7152 & 2015.280 & $\mathrm{R}$ & 20 & 1.100 & 0.020 & $268.8^{*}$ & * 0.3 & & & & \\
\hline $09035+3750$ & MUG1 & - & 2015.304 & $\mathrm{R}$ & 20 & - & - & - & - & Too Faint & & & \\
\hline $09036+4709$ & A1585 & 7158 & 2015.214 & $\mathrm{R}$ & 10 & 0.269 & 0.011 & $286.7^{*}$ & * 0.3 & Elongated & Hrt2000a & -0.02 & 0.2 \\
\hline $09051+3931$ & AG160 & 7167 & 2015.285 & $\mathrm{R}$ & 32 & 4.001 & 0.020 & $58.6^{*}$ & * 0.4 & & & & \\
\hline $09057+3227$ & AG161 & 7174 & 2015.304 & W & 32 & 4.394 & 0.060 & $224.2^{*}$ & * 1.1 & & & & \\
\hline $09071+3037$ & AG162 & 7183 & 2015.285 & $\mathrm{R}$ & 32 & 4.073 & 0.035 & $105.5^{*}$ & * 0.3 & & & & \\
\hline $09095+0256$ & STT197 & 7215 & 2015.285 & $\mathrm{R}$ & 32 & 1.379 & 0.017 & $67.6^{*}$ & * 0.8 & & & & \\
\hline $09103+5223$ & STF1312 & 7212 & 2015.214 & $\mathrm{R}$ & 32 & 4.674 & 0.023 & $148.1^{*}$ & * 0.3 & & & & \\
\hline $09104+6708$ & STF1306 & 7203 & 2015.214 & $\mathrm{RL}$ & 32 & 4.421 & 0.039 & $348.1^{*}$ & * 0.3 & Diffuse & Sca2015b & 0.08 & 0.1 \\
\hline $09149+0413$ & BU455 & 7257 & 2015.302 & $\mathrm{R}$ & 32 & 1.697 & 0.028 & $67.1^{*}$ & * 0.7 & & & & \\
\hline $09179+2834$ & STF3121 & 7284 & 2015.307 & $\mathrm{R}$ & 10 & 0.308 & 0.005 & 190.6 & 1.0 & & Sod1999 & -0.02 & $0.6^{Q}$ \\
\hline $09184+3522$ & STF1333 & 7286 & 2015.307 & $\mathrm{R}$ & 20 & 1.931 & 0.010 & $49.2^{*}$ & * 0.3 & & & & \\
\hline $09210+3811$ & STF1338AB & 7307 & 2015.272 & $\mathrm{R}$ & 20 & 1.109 & 0.008 & $308.3^{*}$ & * 0.3 & & Sca2002b & 0.10 & -5.2 \\
\hline $09235+3908$ & STF1344 & 7332 & 2015.272 & $\mathrm{R}$ & 32 & 3.787 & 0.019 & $102.6^{*}$ & * 0.4 & & & & \\
\hline $09270+6421$ & STF1345 & 7364 & 2015.304 & W & 32 & 2.836 & 0.023 & $85.0^{*}$ & * 0.3 & & & & \\
\hline $09273+0614$ & STF1355 & 7380 & 2015.272 & $\mathrm{R}$ & 20 & 1.789 & 0.009 & 354.6 & 0.3 & & Lin2011b & -0.02 & -0.1 \\
\hline $09277+1545$ & STF1353 & 7386 & 2015.285 & W & 32 & 3.249 & 0.017 & 124.5 & 0.3 & & & & \\
\hline $09315+0128$ & STF1365 & 7412 & 2015.302 & $\mathrm{R}$ & 32 & 3.383 & 0.017 & $156.1^{*}$ & * 0.3 & Diffuse & & & \\
\hline $09414+3857$ & STF1374 & 7477 & 2015.288 & $\mathrm{R}$ & 32 & 2.801 & 0.023 & $309.4^{*}$ & * 0.3 & & Lin2013a & -0.01 & -1.2 \\
\hline $09521+1628$ & STF1390AB & 7549 & 2015.310 & W & 32 & 1.949 & 0.045 & $208.0^{*}$ & * 0.6 & & & & \\
\hline$"$ & SE5AC & - & 2015.310 & W & 32 & 11.276 & 0.056 & $40.7^{*}$ & * 0.3 & & & & \\
\hline $09524+2659$ & STF1389 & 7551 & 2015.288 & $\mathrm{R}$ & 32 & 2.496 & 0.045 & $289.4^{*}$ & * 0.3 & & Lin2016a & -0.01 & -0.8 \\
\hline $09556+0806$ & AG170 & 7579 & 2015.302 & $\mathrm{R}$ & 32 & 1.610 & 0.060 & $44.6^{*}$ & * 1.4 & & & & \\
\hline $09564+1040$ & STF1396 & 7585 & 2015.310 & W & 32 & 3.877 & 0.019 & $129.1^{*}$ & * 0.3 & & & & \\
\hline $10057+4103$ & A 2142 & 7631 & 2015.307 & $\mathrm{R}$ & 20 & 1.086 & 0.015 & $293.8^{*}$ & * 0.5 & & & & \\
\hline $10123+1621$ & STF1413 & 7679 & 2015.302 & W & 32 & 1.664 & 0.067 & 270.5 & 0.6 & & & & \\
\hline $10131+2725$ & STT213 & 7685 & 2015.280 & $\mathrm{R}$ & 20 & 1.124 & 0.008 & 121.0 & 0.6 & & Sca2008e & 0.05 & 0.3 \\
\hline $10151+1907$ & STF1417 & 7695 & 2015.302 & W & 32 & 2.208 & 0.080 & 75.4 & 0.7 & & & & \\
\hline $10163+1744$ & STT215 & 7704 & 2015.272 & $\mathrm{R}$ & 20 & 1.465 & 0.008 & $176.3^{*}$ & * 0.3 & & Zae1984 & -0.09 & -2.1 \\
\hline $10181+2731$ & STF1421 & 7715 & 2015.307 & $\mathrm{R}$ & 32 & 4.598 & 0.023 & $330.9^{*}$ & * 0.3 & & & & \\
\hline $10200+1950$ & STF1424AB & 7724 & 2015.285 & $\mathrm{R}$ & 32 & 4.729 & 0.033 & $125.6^{*}$ & * 0.4 & & WSI2006b & -0.10 & -0.6 \\
\hline $10205+0626$ & STF1426AB & 7730 & 2015.272 & $\mathrm{R}$ & 20 & 0.896 & 0.008 & 312.1 & 0.3 & & Sca2006b & -0.00 & -0.8 \\
\hline$"$ & STF1426BC & 7730 & 2015.272 & $\mathrm{R}$ & 32 & 7.397 & 0.037 & $14.6^{*}$ & * 0.3 & & & & \\
\hline$"$ & STF1426AC & 7730 & 2015.272 & $\mathrm{R}$ & 32 & 7.821 & 0.039 & $8.8^{*}$ & * 0.3 & & & & \\
\hline$"$ & STF1426AB-C & 7730 & 2015.272 & $\mathrm{R}$ & 32 & 7.657 & 0.038 & $10.9^{*}$ & * 0.3 & & & & \\
\hline $10250+2437$ & STF1429 & 7758 & 2015.280 & $\mathrm{R}$ & 20 & 0.804 & 0.009 & 156.5 & 0.4 & & Zul1981 & 0.05 & -0.2 \\
\hline $10260+5237$ & STF1428 & 7762 & 2015.307 & $\mathrm{R}$ & 32 & 2.775 & 0.044 & $86.0^{*}$ & * 0.3 & & & & \\
\hline $10269+1713$ & STT217 & 7775 & 2015.304 & $\mathrm{R}$ & 20 & 0.726 & 0.009 & $151.1^{*}$ & * 0.5 & & Sca2015b & -0.06 & 2.6 \\
\hline $10480+4107$ & STT229 & 7929 & 2015.280 & $\mathrm{R}$ & 20 & 0.646 & 0.008 & $260.0^{*}$ & * 0.4 & & Alz1998a & 0.01 & 3.0 \\
\hline $10556+2445$ & STF1487 & 7979 & 2015.302 & $\mathrm{R}$ & 32 & 6.626 & 0.033 & $111.9^{*}$ & * 0.3 & Elongated & & & \\
\hline $10557+0044$ & BU1076 & 7982 & 2015.376 & $\mathrm{R}$ & 20 & 1.111 & 0.011 & $52.1^{*}$ & * 0.5 & & Tok2015c & -0.01 & 0.4 \\
\hline
\end{tabular}


Table 1 Table of speckle measurements and O-C residuals with published orbits (cont.)

\begin{tabular}{|c|c|c|c|c|c|c|c|c|c|c|c|c|}
\hline WDS & Name & $\mathrm{ADS}$ & Epoch & Fil. & $\begin{array}{l}\text { Eyep. } \\
(\mathrm{mm})\end{array}$ & $\begin{array}{c}\rho \\
\left(^{\prime \prime}\right)\end{array}$ & $\begin{array}{l}\sigma_{\rho} \\
\left({ }^{\prime \prime}\right)\end{array}$ & $\begin{array}{cc}\theta & \sigma_{\theta} \\
\left(^{\circ}\right) & \left(^{\circ}\right)\end{array}$ & Notes & Orbit & $\begin{array}{r}\Delta \rho(\mathrm{O}-\mathrm{C}) \\
\quad\left({ }^{\prime \prime}\right)\end{array}$ & $\begin{array}{r}\Delta \theta(\mathrm{O}-\mathrm{C}) \\
\left({ }^{\circ}\right)\end{array}$ \\
\hline $11035+5432$ & A1590 & 8032 & 2015.285 & $\mathrm{R}$ & 32 & 1.415 & 0.033 & $332.9^{*} 1.3$ & & Baz1985b & -0.09 & 1.1 \\
\hline $11037+6145$ & BU1077 & 8035 & 2015.280 & $\mathrm{R}$ & 10 & 0.736 & 0.004 & $350.1^{*} 0.3$ & & Sca2011a & -0.00 & -2.5 \\
\hline$"$ & $"$ & & 2015.376 & $\mathrm{R}$ & 10 & 0.749 & 0.011 & $350.0^{*} 0.4$ & & Sca2011a & 0.01 & -2.1 \\
\hline $11137+2008$ & STF1517 & 8094 & 2015.307 & $\mathrm{R}$ & 20 & 0.660 & 0.009 & 318.31 .0 & & FMR2015b & -0.04 & 2.2 \\
\hline $11182+3132$ & STF1523AB & 8119 & 2015.305 & $\mathrm{R}$ & 20 & 1.732 & 0.009 & $175.0^{*} 0.3$ & & Sod1999 & -0.01 & -0.1 \\
\hline $11190+1416$ & STF1527 & 8128 & 2015.280 & $\mathrm{R}$ & 10 & 0.307 & 0.003 & $251.4^{*} 0.7$ & & Tok2012b & 0.01 & 0.3 \\
\hline $11239+1032$ & STF1536 & 8148 & 2015.285 & $\mathrm{R}$ & 20 & 2.120 & 0.011 & $95.9^{*} 0.3$ & & Sod1999 & 0.01 & 0.2 \\
\hline $11323+6105$ & STT235 & 8197 & 2015.403 & $\mathrm{R}$ & 20 & 0.883 & 0.008 & $38.2^{*} 1.2$ & & Sod1999 & -0.01 & 2.3 \\
\hline $11347+1648$ & STF1552 & 8220 & 2015.307 & $\mathrm{R}$ & 32 & 3.556 & 0.018 & $208.8^{*} 0.3$ & Diffuse & & & \\
\hline $11363+2747$ & STF1555 & 8231 & 2015.305 & $\mathrm{R}$ & 20 & 0.740 & 0.011 & $148.6^{*} 0.7$ & & Doc2017a & 0.05 & -1.3 \\
\hline $11390+4109$ & STT237 & 8252 & 2015.305 & $\mathrm{R}$ & 32 & 2.062 & 0.030 & $242.6^{*} 0.4$ & & USN2002 & 0.03 & -1.7 \\
\hline $11406+2102$ & STF1566 & 8263 & 2015.419 & $\mathrm{R}$ & 32 & 2.298 & 0.018 & $349.0^{*} 0.8$ & & & & \\
\hline $11563+3527$ & STT241 & 8355 & 2015.425 & $\mathrm{R}$ & 20 & 1.781 & 0.009 & $145.7^{*} 0.3$ & & & & \\
\hline $12108+3953$ & STF1606 & 8446 & 2015.403 & $\mathrm{R}$ & 20 & 0.549 & 0.008 & $149.3^{*} 1.1$ & & Msn1999a & -0.01 & 3.8 \\
\hline $12126+3546$ & STF1613 & 8460 & 2015.403 & $\mathrm{R}$ & 32 & 1.132 & 0.037 & $\begin{array}{lll}7.2 & 0.7\end{array}$ & & & & \\
\hline $12160+0538$ & STF1621 & 8486 & 2015.425 & W & 32 & 1.520 & 0.025 & $44.6^{*} 0.9$ & & Sod1999 & -0.14 & -0.4 \\
\hline $12244+2535$ & STF1639 & 8539 & 2015.403 & $\mathrm{R}$ & 20 & 1.818 & 0.023 & $322.8^{*} 0.6$ & & Ole2000b & -0.01 & -0.3 \\
\hline $12272+2701$ & STF1643 & 8553 & 2015.419 & $\mathrm{R}$ & 32 & 2.889 & 0.025 & $4.7^{*} 0.3$ & & WSI2004a & 0.14 & 0.8 \\
\hline $12417-0127$ & STF1670 & 8630 & 2015.280 & $\mathrm{R}$ & 20 & 2.270 & 0.016 & $\begin{array}{lll}5.3 & 0.3\end{array}$ & & Sca2007c & -0.04 & -0.1 \\
\hline$"$ & $"$ & $"$ & 2015.376 & $\mathrm{R}$ & 20 & 2.300 & 0.012 & $\begin{array}{ll}4.9 & 0.3\end{array}$ & & Sca2007c & -0.02 & -0.2 \\
\hline $13007+5622$ & BU1082 & 8739 & 2015.376 & $\mathrm{R}$ & 20 & 0.889 & 0.014 & $116.5^{*} 0.4$ & & Sca2012c & 0.01 & -0.5 \\
\hline $13084+1529$ & STF1722 & 8796 & 2015.425 & $\mathrm{R}$ & 32 & 2.628 & 0.028 & $336.0^{*} 0.3$ & & & & \\
\hline $13120+3205$ & STT261 & 8814 & 2015.425 & $\mathrm{R}$ & 32 & 2.578 & 0.017 & $337.6^{*} 0.3$ & & Kis2012 & -0.01 & -0.8 \\
\hline $13166+5034$ & STT263 & 8843 & 2015.419 & $\mathrm{R}$ & 32 & 1.766 & 0.030 & $135.8 \quad 0.9$ & & & & \\
\hline $13284+1543$ & STT266 & 8914 & 2015.420 & $\mathrm{R}$ & 32 & 1.945 & 0.017 & $355.6^{*} 0.6$ & & Hrt2011d & -0.05 & -2.0 \\
\hline $13324+3649$ & STF1755 & 8934 & 2015.425 & $\mathrm{R}$ & 32 & 4.123 & 0.022 & $129.3^{*} 0.3$ & & & & \\
\hline $13329+4908$ & STF1758 & 8940 & 2015.420 & $\mathrm{R}$ & 32 & 3.316 & 0.023 & $291.1^{*} 0.3$ & & & & \\
\hline $13346+3308$ & BU933AB & 8958 & 2015.425 & $\mathrm{~W}$ & 32 & 2.781 & 0.017 & $21.9^{*} 0.6$ & Diffuse & & & \\
\hline $13491+2659$ & STF1785 & 9031 & 2015.425 & $\mathrm{R}$ & 32 & 2.993 & 0.018 & $186.3^{*} 0.5$ & & Hei1988d & 0.08 & 0.2 \\
\hline $14165+2007$ & STF1825 & 9192 & 2015.425 & $\mathrm{R}$ & 32 & 4.384 & 0.027 & $152.7^{*} 0.4$ & & Kiy2017 & -0.05 & -0.4 \\
\hline $14203+4830$ & STF1834 & 9229 & 2015.420 & $\mathrm{R}$ & 32 & 1.595 & 0.028 & $102.2^{*} 0.5$ & & WSI2015 & -0.00 & -1.1 \\
\hline $22514+6142$ & STF2950 & 16317 & 2015.016 & $\mathrm{R}$ & 20 & 1.174 & 0.008 & $274.6^{*} 0.3$ & & Zir2015a & -0.01 & -0.1 \\
\hline $22537+4445$ & BU382AB & 16345 & 2015.003 & $\mathrm{R}$ & 20 & 0.735 & 0.010 & $241.5^{*} 0.3$ & & Sca2014a & 0.02 & 1.0 \\
\hline $22542+7620$ & STF2963 & 16371 & 2015.017 & $\mathrm{R}$ & 32 & 1.840 & 0.017 & $2.6^{*} 0.4$ & & & & \\
\hline $22557+1547$ & HU987 & 16373 & 2015.003 & $\mathrm{R}$ & 32 & 1.210 & 0.022 & $76.0^{*} 1.8$ & & USN2007a & 0.08 & -1.2 \\
\hline $23103+3229$ & BU385AB & 16561 & 2015.003 & $\mathrm{R}$ & 20 & 0.658 & 0.010 & $84.7^{*} 0.9$ & & Lin2010c & -0.02 & 0.3 \\
\hline
\end{tabular}

Note: In column $9, *$ indicates that $\theta$ was determined with our quadrant value (or with the long integration).

In column 14, the exponent ${ }^{Q}$ indicates discrepant quadrants between our measurements and the published orbits.

ber in Col. 3 (Aitken, 1932) when available. For each observation, we then give the epoch in Besselian years (Col. 4), the filter (Col. 5), the focal length of the eyepiece used for magnifying the image (Col. 6), the angular separation $\rho$ (Col. 7) with its error (Col. 8) in arcseconds, and the position angle $\theta$ (Col. 9) with its error (Col. 10) in degrees. In Col. 11, we report some notes and some information about the secondary peaks of the autocorrelation files. More precisely, "Elongated" and "Diffuse" are related to those peaks only and the corresponding observations are fully resolved, unless explicitly mentioned by "Unresolved" in this column.
For the systems with a known orbit, the $(O-C)$ (Observed minus Computed) residuals of the $\rho$ and $\theta$ measurements are displayed in Cols. 13 and 14, respectively. The corresponding authors are given in Col. 12, using the bibliographic style of the "Sixth Catalog of Orbits of Visual Binary Stars" (Hartkopf \& Mason, 2018, hereafter OC6).

When not explicitly specified, the measurements refer to the $\mathrm{AB}$ components of those systems. In Col. 14, the symbol $Q$ indicates that there was a quadrant inconsistency between our measures and the positions de- 


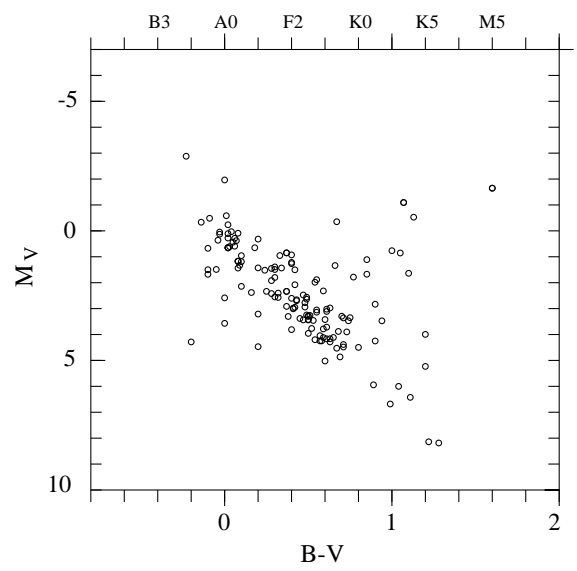

Fig. 4 HR diagram of the binaries measured in Table 1, for which Hipparcos parallaxes were obtained with a relative error smaller than $50 \%$ (i.e., 146 objects).

rived from the orbital elements published for this object.

The characteristics of the $\mathrm{R}$ and RL filters used for obtaining those measurements are given in Table 1 of Paper XII. Some objects were observed without any filter because they were too faint. This is indicated with W (for "white" light) in the filter column (Col. 5 of Table 1). In that case, the bandpass and central wavelength correspond to that of the ICCD detector (see Prieur et al., 1998).

As for the other papers of this series, position measurements were obtained by an interactive processing of the autocorrelation files computed in real time during the observations. This processing led to a series of measurements with different background estimates and simulated noise, from which we derived the mean values and the standard deviation of those multiple measurements (see Paper III for more details). The final measures and their errors are displayed in Table 1. The average error values of the measurements reported in this table are $\left\langle\sigma_{\rho}\right\rangle=0^{\prime \prime} .019 \pm 0^{\prime \prime} .015$ and $\left\langle\sigma_{\theta}\right\rangle=0^{\circ} .6 \pm 1^{\circ} .5$ for the angular separation $\rho$ and the position angle $\theta$, respectively.

\subsection{Comparison between PISCO and Gaia relative astrometry}

The second release of Gaia measurements (ESA, 2018) has provided astrometric measurements of some of our measured pairs at 2015.5, close to the date of the observations. We have thus been able to compare the measurements of 110 pairs observed by PISCO (from Table 1) with the corresponding entries in Gaia DR2.

The mean errors and standard deviations for $\rho$ and $\theta$ are $-0^{\prime \prime} .0051 \pm 0.05$ and $-0^{\circ} .53 \pm 1^{\circ} .26$, respectively. The plots in $\rho$ and $\theta$ do not show any deviance from
Table 2 Gaia versus PISCO relative astrometry: objects with the largest differences, i.e., $\Delta \rho>0^{\prime \prime} .1$ or $\Delta \theta>4^{\circ}$.

\begin{tabular}{|c|c|c|c|c|c|}
\hline \multirow{2}{*}{$\begin{array}{l}\text { HIP } \\
\text { (A comp.) }\end{array}$} & \multirow[t]{2}{*}{ Name } & \multicolumn{2}{|c|}{ PISCO } & \multicolumn{2}{|c|}{ Gaia-DR2 } \\
\hline & & $\begin{array}{l}\rho \\
\left({ }^{\prime \prime}\right)\end{array}$ & $\begin{array}{c}\theta \\
\left({ }^{\circ}\right)\end{array}$ & $\begin{array}{l}\rho \\
\left({ }^{\prime \prime}\right)\end{array}$ & $\begin{array}{c}\theta \\
\left({ }^{\circ}\right)\end{array}$ \\
\hline HIP1670 & BU 1093 & 0.752 & 117.6 & 0.745 & 121.5 \\
\hline HIP15482 & HU 1056 & 1.200 & 77.7 & 1.079 & 79.6 \\
\hline HIP31345 & STF 932 & 1.580 & 300.8 & 1.653 & 305.2 \\
\hline HIP 54040 & A 1590 & 1.415 & 332.9 & 1.467 & 328.4 \\
\hline HIP56601 & STF 1555 & 0.740 & 148.6 & 0.612 & 155.8 \\
\hline HIP59816 & STF1621 & 1.520 & 44.6 & 1.666 & 45.6 \\
\hline HIP 60579 & STF 1643 & 2.889 & 4.7 & 2.753 & 4.2 \\
\hline HIP63503 & BU 1082 & 0.889 & 116.5 & 0.849 & 121.3 \\
\hline
\end{tabular}
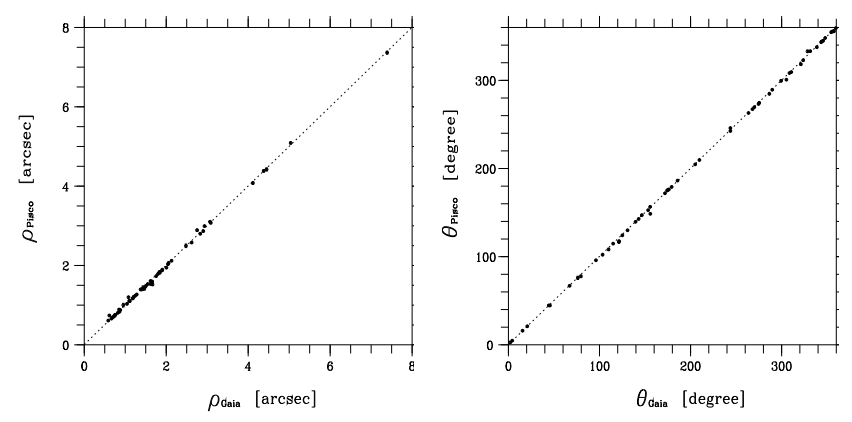

Fig. 5 Comparison between PISCO and Gaia relative astrometry.

linearity between PISCO and Gaia (Figs. 5). The agreement between PISCO and Gaia-DR2 relative astrometry is therefore very good in general.

However, there are some cases with significant discrepancies in $\rho$ and $\theta$, that are reported in Table 2 , with differences in $\rho$ and $\theta$ measurements as large as $0 .^{\prime \prime} 1$ and $4^{\circ}$, respectively. Such differences may be due to the fact that there was not any dedicated data-reduction program for measuring close binaries in DR2. This should come later with the future releases of Gaia measurements. In the mean time, it seems that ground-based speckle observations remain the most reliable source of information for close binary relative astrometry.

Note also that the parallaxes for the two components of STF $1126=$ ADS 6263 are different $(\mathrm{A}=$ 4.96 mas $+/-0.22, \mathrm{~B}=2.33$ mas $+/-0.37)$, which is puzzling since the orbital motion of this pair is well established by the observations.

\subsection{Quadrant determination}

As our astrometric measurements were obtained from the symmetric autocorrelation files, the $\theta$ values first presented a $180^{\circ}$ ambiguity. To resolve this ambiguity and determine the quadrant containing the companion, Aristidi et al. (1997) have proposed a method that can 
be considered as a restricted triple correlation (RTC hereafter). The quadrants of the measurements indicated in Table 1 were mostly derived from the RTC files that were computed in real time during the observations. However, for the couples with the largest separations, a straightforward determination was done when the companions could be directly spotted on the long integration files.

As a result, in Table 1, we are able to give the unambiguous (i.e. "absolute") position angles of 159 out of 196 measurements, i.e. $81 \%$ of the total. They are marked with an asterisk in Col 9. When our quadrant determination procedure failed, the angular measurement was reverted to the quadrant reported in the WDS catalog, which is extracted from the Fourth Catalog of Interferometric Measurements of Binary Stars (Hartkopf et al. 2018, hereafter IC4).

Our "absolute" $\theta$ values are consistent with the values tabulated in WDS for all objects except for ADS 3353. This determination was clear from our observations, since we directly saw the companion in the first quadrant (North-East). We already found such a result in March 2012 with PISCO (Paper XIII). The origin of this discrepancy may be due to the small difference of magnitude $\Delta m_{V}=0.15$ between the two components, and the fact that our observations were made in $R$, whereas the other observers used a $B$ filter. Moreover, a different spectral type of the two components could also account for a quadrant inversion.

\subsection{Comparison with published ephemerides}

The $(O-C)$ (Observed minus Computed) residuals of the measurements for the systems with a known orbit in Table 1 are displayed in Cols. 13 and 14 for the separation $\rho$ and position angle $\theta$, respectively. Those residuals were obtained with a selection of valid orbits found in the OC6 catalog. We did not always use the most recent orbits since sometimes older orbits led to equivalent or even smaller residuals. For ADS 6276 we also report the residuals obtained with our revised orbit presented in Sect. 4.

The residuals are plotted in Fig. 6. They have a rather large scatter which is naturally explained by the (old) age of many orbits that will need revision in the future. The mean values computed with the residuals of Table 1 are $\left\langle\Delta \rho_{O-C}\right\rangle=0^{\prime \prime} .007 \pm 0^{\prime \prime} .05$ and $\left\langle\Delta \theta_{O-C}\right\rangle=-0^{\circ} .2 \pm 1^{\circ} .7$. The small values obtained for those offsets provide a good validation of our calibration (see Paper XII).

\subsection{Rectilinear elements of ADS 4841}

As shown in Fig. 6, large residuals have been found for ADS 4841 (BU 1008 - eta Gem) for our two observations made in 2015, when using the orbit of Baize (1980). We have investigated this problem, and

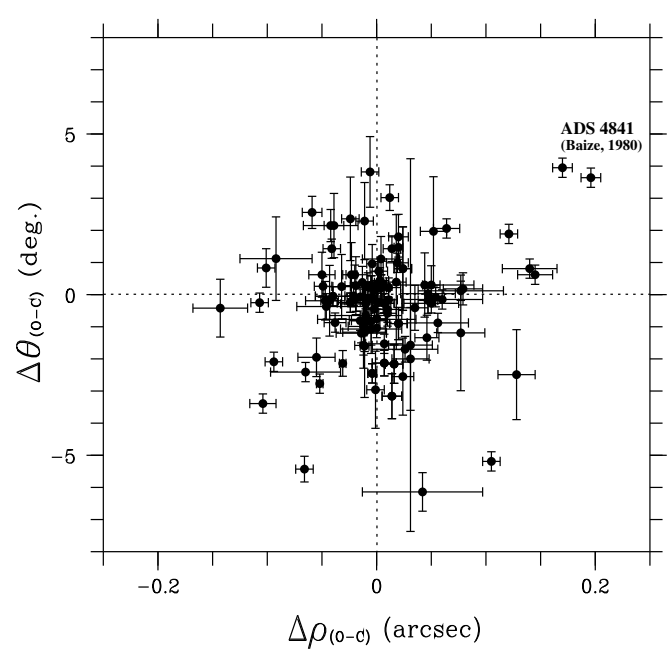

Fig. 6 Residuals of the measurements of Table 1 computed with the published orbits.

tried to improve this orbit. When examining the trajectory of the companion from the 105 observations made since 1881, it appears that the companion is moving along a straight line relative to the primary star, within the error bars (see Fig. 7). This linear trajectory seems to indicate that the two components are not gravitationally linked, which may also be corroborated by the large brightness difference of $\Delta m_{V}=2.6$ between the two components. We hope that the future parallax measurements by Gaia will allow to answer to this question. In the meantime, we have computed a rectilinear trajectory that well accounts for the observations (see Table 4 of the residuals with Julian epochs since 2004). Following the WDS convention, we fitted the relative Cartesian coordinates $(\mathrm{X}, \mathrm{Y})$ of the companion with the model:

$$
\begin{aligned}
& \mathrm{X}=\mathrm{XA} *(\mathrm{~T}-\mathrm{T} 0)+\mathrm{X} 0 \\
& \mathrm{Y}=\mathrm{YA} *(\mathrm{~T}-\mathrm{T} 0)+\mathrm{Y} 0
\end{aligned}
$$

where $\mathrm{T}$ is the epoch, and (X0, Y0, T0) is the relative location and epoch of closest approach. We used Julian epochs for this fit. The corresponding rectilinear elements are given in Table 3, where we also report the polar coordinates (RH0, THETA0) corresponding to that closest approach, i.e.,

$$
\begin{aligned}
& \mathrm{X} 0=-\sin (\text { THETA0 }) * \text { RHO0 } \\
& \mathrm{Y0}=\cos (\text { THETA0 }) * \text { RHO0 }
\end{aligned}
$$

The ephemerides of ADS 4841 for 2018-2028 are presented in Table 6 . 
Table 5 New orbital elements of DUN 5, ADS 5958, 6276, 7294, 8211 and 13169.

\begin{tabular}{|c|c|c|c|c|c|c|c|c|c|c|c|c|}
\hline Name & $\begin{array}{c}\Omega_{2000} \\
\left(^{\circ}\right)\end{array}$ & $\begin{array}{l}\omega \\
\left(^{\circ}\right)\end{array}$ & $\begin{array}{c}i \\
\left(^{\circ}\right)\end{array}$ & $e$ & $\begin{array}{c}T \\
(\mathrm{yr})\end{array}$ & $\begin{array}{c}P \\
(\mathrm{yr})\end{array}$ & $\begin{array}{c}n \\
(\% / y r)\end{array}$ & $\begin{array}{c}a \\
\left({ }^{\prime \prime}\right)\end{array}$ & $\begin{array}{c}\mathrm{A} \\
\left({ }^{\prime \prime}\right)\end{array}$ & $\begin{array}{c}\mathrm{B} \\
\left({ }^{\prime \prime}\right)\end{array}$ & $\begin{array}{c}\mathrm{F} \\
\left({ }^{\prime \prime}\right)\end{array}$ & $\begin{array}{l}\mathrm{G} \\
\left({ }^{\prime \prime}\right)\end{array}$ \\
\hline DUN 5 & $\begin{array}{c}16.50 \\
-\end{array}$ & $\begin{array}{c}24.90 \\
-\end{array}$ & $\begin{array}{c}139.6 \\
-\end{array}$ & $\begin{array}{c}0.499 \\
-\end{array}$ & $\begin{array}{c}1811.9 \\
-\end{array}$ & $\begin{array}{c}493.3 \\
-\end{array}$ & $\begin{array}{c}0.7297 \\
-\end{array}$ & $\begin{array}{c}7.98 \\
-\end{array}$ & 7.66684 & -0.39754 & -1.65596 & -6.23944 \\
\hline ADS 5958 & $\begin{array}{l}93.30 \\
\pm 0.75\end{array}$ & $\begin{array}{l}124.7 \\
\pm 6.1\end{array}$ & $\begin{array}{l}109.5 \\
\pm 0.7\end{array}$ & $\begin{array}{c}0.520 \\
\pm 0.008\end{array}$ & $\begin{array}{c}2015.27 \\
\pm 0.94\end{array}$ & $\begin{array}{l}289.2 \\
\pm 17\end{array}$ & $\begin{array}{l}1.2447 \\
\pm 0.074\end{array}$ & $\begin{array}{c}1.366 \\
\pm 0.026\end{array}$ & 0.41902 & -0.75477 & -0.19450 & -1.13613 \\
\hline ADS 6276 & $\begin{array}{c}60.70 \\
-\end{array}$ & $\begin{array}{c}105.60 \\
-\end{array}$ & $\begin{array}{c}180.0 \\
-\end{array}$ & $\begin{array}{c}0.792 \\
-\end{array}$ & $\begin{array}{c}1956.64 \\
-\end{array}$ & $\begin{array}{c}174.0 \\
-\end{array}$ & $\begin{array}{c}2.0689 \\
-\end{array}$ & $\begin{array}{c}0.334 \\
-\end{array}$ & 0.23659 & -0.23576 & -0.23576 & -0.23659 \\
\hline ADS 7294 & $\begin{array}{c}60.20 \\
-\end{array}$ & $\begin{array}{c}203.7 \\
-\end{array}$ & $\begin{array}{c}139.9 \\
-\end{array}$ & $\begin{array}{c}0.664 \\
-\end{array}$ & $\begin{array}{c}1943.74 \\
-\end{array}$ & $\begin{array}{c}820.2 \\
-\end{array}$ & $\begin{array}{c}0.4389 \\
-\end{array}$ & $\begin{array}{c}0.905 \\
-\end{array}$ & -0.65329 & -0.58081 & -0.36927 & 0.63068 \\
\hline ADS 8211 & 11.50 & $\begin{array}{c}95.6 \\
-\end{array}$ & $\begin{array}{c}55.6 \\
-\end{array}$ & $\begin{array}{c}0.487 \\
-\end{array}$ & $\begin{array}{c}1871.40 \\
-\end{array}$ & $\begin{array}{c}373.7 \\
-\end{array}$ & $\begin{array}{c}0.9635 \\
-\end{array}$ & $\begin{array}{c}0.645 \\
-\end{array}$ & -0.13398 & 0.34284 & -0.62195 & -0.16282 \\
\hline ADS 13169 & $\begin{array}{c}107.50 \\
-\end{array}$ & $\begin{array}{c}251.3 \\
-\end{array}$ & 12.5 & $\begin{array}{c}0.379 \\
-\end{array}$ & $\begin{array}{c}1924.46 \\
-\end{array}$ & $\begin{array}{c}220.8 \\
-\end{array}$ & $\begin{array}{c}1.6301 \\
-\end{array}$ & $\begin{array}{c}0.410 \\
-\end{array}$ & 0.40113 & -0.01135 & 0.00561 & 0.40897 \\
\hline
\end{tabular}

Table 6 New ephemerides of DUN 5, ADS 4841, 5958, 6276, 7294 and 13169.

\begin{tabular}{|c|c|c|c|c|c|c|c|c|c|c|c|c|c|c|}
\hline \multirow[b]{2}{*}{ Epoch } & \multicolumn{2}{|c|}{ DUN 5} & \multicolumn{2}{|c|}{ ADS 4841} & \multicolumn{2}{|c|}{ ADS 5958} & \multicolumn{2}{|c|}{ ADS 6276} & \multicolumn{2}{|c|}{ ADS 7294} & \multicolumn{2}{|c|}{ ADS 8211} & \multicolumn{2}{|c|}{ ADS 13169} \\
\hline & $\begin{array}{c}\rho \\
\left(^{\prime \prime}\right)\end{array}$ & $\begin{array}{c}\theta \\
\left({ }^{\circ}\right)\end{array}$ & $\begin{array}{c}\rho \\
\left(^{\prime \prime}\right)\end{array}$ & $\begin{array}{c}\theta \\
\left(^{\circ}\right)\end{array}$ & $\begin{array}{c}\rho \\
\left(^{\prime \prime}\right)\end{array}$ & $\begin{array}{c}\theta \\
\left({ }^{\circ}\right)\end{array}$ & $\begin{array}{c}\rho \\
\left({ }^{\prime \prime}\right)\end{array}$ & $\begin{array}{c}\theta \\
\left(^{\circ}\right)\end{array}$ & $\begin{array}{c}\rho \\
\left({ }^{\prime \prime}\right)\end{array}$ & $\begin{array}{c}\theta \\
\left({ }^{\circ}\right)\end{array}$ & $\begin{array}{c}\rho \\
\left(^{\prime \prime}\right)\end{array}$ & $\begin{array}{c}\theta \\
\left({ }^{\circ}\right)\end{array}$ & $\begin{array}{c}\rho \\
\left(^{\prime \prime}\right)\end{array}$ & $\begin{array}{c}\theta \\
\left({ }^{\circ}\right)\end{array}$ \\
\hline 2018.0 & 11.593 & 186.3 & 1.855 & 256.6 & 0.508 & 290.5 & 0.563 & 145.6 & 0.607 & 96.5 & 0.540 & 264.1 & 0.547 & 164.6 \\
\hline 019 . & .599 & 186.0 & 1.862 & 256.5 & 0.541 & 38.0 & 0.565 & 145.2 & 0.614 & 95.9 & 0.540 & 264.7 & 0.547 & 165.5 \\
\hline 2020.0 & 1.604 & 185.8 & 1.870 & 256.3 & 0.572 & 285.9 & 0.568 & 144.7 & 0.620 & 95.4 & 0.539 & 265.4 & 0.548 & 166.3 \\
\hline 2021.0 & 1.609 & 185 & 1.878 & 256.2 & 0.602 & 34.0 & 0.571 & 144.3 & 0.627 & 94.9 & 0.539 & 266.1 & 0.549 & 167.1 \\
\hline 2 & .613 & 5.4 & 1.886 & 256.0 & 0.630 & 282.2 & 0.573 & 143.9 & 0.634 & 94.3 & 0.539 & 266.8 & 0.550 & 167.9 \\
\hline 2023.0 & 1.617 & 185.1 & 1.894 & 255.9 & 0.656 & 280.6 & 0.575 & 143.4 & 0.640 & 93.8 & 0.539 & 267.5 & 0.550 & 168.7 \\
\hline 2024.0 & 11.621 & 184.9 & 1.901 & 255.7 & 0.680 & 279.1 & 0.578 & 143.0 & 0.647 & 93.3 & 0.538 & 268.1 & 0.551 & 169.6 \\
\hline & 11.624 & & 1.909 & 255.6 & 0.703 & 277.8 & 0.580 & 142.6 & 0.653 & 92.9 & 0.538 & 268.8 & 0.552 & 170.4 \\
\hline 2026.0 & 11.627 & & 1.917 & 255.5 & 0.723 & 276.5 & 0.582 & 142.2 & 0.660 & 92.4 & 0.538 & 269.5 & 0.552 & 171.2 \\
\hline 2027.0 & 11.630 & 184.3 & 1.925 & 255.3 & 0.742 & 275.2 & 0.584 & 141.8 & 0.666 & 91.9 & 0.538 & 270.2 & 0.552 & 172.0 \\
\hline 2028.0 & 11.632 & 184.0 & 1.933 & 255.2 & 0.759 & 274.1 & 0.585 & 141.3 & 0.672 & 91.5 & 0.538 & 270.9 & 0.553 & 172.8 \\
\hline
\end{tabular}

Table 3 New rectilinear elements of ADS 4841.

$\mathrm{X} 0=-0.7054200 \pm 0.0229 \mathrm{XA}=-0.0064235 \pm 0.0002$ $\mathrm{Y} 0=-0.6916823 \pm 0.0184 \mathrm{YA}=+0.0065519 \pm 0.0002$ RO0 $=0^{\prime \prime} .988 \quad$ TETA0 $=314^{\circ} .44 \quad$ T0 $=1846.926$

\section{Revised orbits of DUN 5, ADS 5958, $6276,7294,8211$ and 13169}

In this section we present the new revised orbits that we have computed for DUN 5, ADS 5958, 6276, 7294, 8211 and 13169. Those objects belong to our list of regularly monitored couples with PISCO in the Northern hemisphere and by one of us (Ary) in the Southern hemisphere. The revision of those orbits was justified by the appearance of a systematic trend in the residuals of our last measurements and/or the existence of substantial number of new measurements since the computation of the last known orbit.

We have followed the same method for computing the orbits of those six objects. Using our last measurements with PISCO and the other available ob-
Table 4 ADS 4841: O-C residuals with our new rectilinear elements (after 2004). The symbol ${ }^{P}$ indicates PISCO measurements.

\begin{tabular}{crcl}
\hline Epoch & $\Delta \rho(\mathrm{O}-\mathrm{C})$ \\
& $\left(^{\prime \prime}\right)$ & $\Delta \theta(\mathrm{O}-\mathrm{C})$ & Observer \\
$\left(^{\circ}\right)$ & \\
\hline 2004.240 & 0.016 & $-0.3^{P}$ & Sca \\
2005.110 & -0.053 & $-1.5^{P}$ & Sca \\
2006.127 & -0.113 & -2.3 & Orl \\
2008.242 & 0.007 & $-1.0^{P}$ & Pru \\
2012.018 & -0.138 & 1.9 & Ary \\
2015.210 & -0.031 & $-0.8^{P}$ & Sca \\
2015.212 & -0.057 & $-0.5^{P}$ & Sca \\
2016.112 & 0.007 & -0.4 & Wss \\
\hline
\end{tabular}

servations contained in the data base maintained by the United States Naval Observatory (USNO), we first computed the preliminary orbital elements with the analytical method of Kowalsky (1873). We then used them as initial values for the least-squares method of Hellerich (1925). When convergence was achieved, Hellerich's method led to an improvement of the orbital elements (with the exception of the major axis) and 


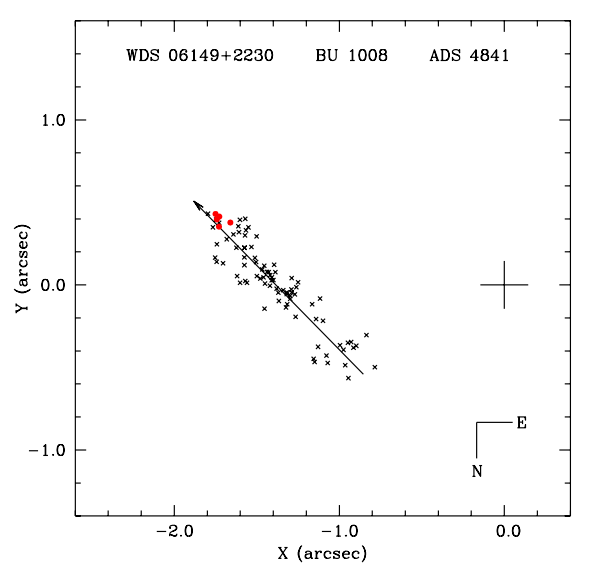

Fig. 7 ADS 4841: relative motion of the companion and plot of our new rectilinear trajectory. The observations by PISCO are plotted as filled circles that appear in red in the electronic version of this paper.

to an estimation of the corresponding errors. The final value of the major axis was then set to the value that minimized the residuals in separation of Hellerich's solution.

The final orbital elements are presented in Table 5, with the periastron passage $T$ (Col. 6) in Julian epoch. The errors reported in this table for ADS 5958 were obtained by Hellerich's least-squares method. For the other objects, Hellerich's method did not converge, which is a good indicator that the overall quality of the data does not allow computing reliable error values. Although other methods (Gauss-Newton or LevenbergMaquard for instance) always provide error values, our long-term experience has shown that those values were not reliable when the quality of the data was not good enough for Hellerich's method to converge. We thus prefer not publishing the errors when the orbits have been not yet sufficiently monitored.

The format of the tables contained in this section is self-explanatory, but a detailed description of those formats can be found in Papers VI and VII.

The ephemerides for 2018-2028 are presented in Table 6 . The apparent orbits are shown in Fig. 8 as solid lines. The observational data used for the calculation of the orbital elements are plotted as small crosses or, in the case of PISCO observations, as filled circles (that appear in red in the electronic version). The orientation of the graphs conforms to the convention adopted by the observers of visual binary stars. For each object, the location of the primary component is indicated with a big cross. The dashed straight line going through this point is the line of apsides. An arrow shows the sense of rotation of the companion.

The $(O-C)$ residuals of the new orbits, restricted to the last observations for reasons of space, are given in Tables 7, 8, 9, 10, 11, 12 for DUN 5, ADS 5958,
Table 7 DUN 5: O-C residuals of our new orbit (after 2009).

\begin{tabular}{rrrl}
\hline Epoch & $\Delta \rho(\mathrm{O}-\mathrm{C})$ \\
$\left({ }^{\prime \prime}\right)$ & $\begin{array}{r}\Delta \theta(\mathrm{O}-\mathrm{C}) \\
\left({ }^{\circ}\right)\end{array}$ & Observer \\
\hline 2009.710 & 0.050 & -0.2 & Ant \\
2009.714 & 0.070 & -0.2 & Ant \\
2010.636 & 0.012 & -0.4 & Ary \\
2013.672 & -0.184 & -0.0 & Ary \\
2013.710 & -0.174 & -0.0 & Ant \\
2016.697 & -0.135 & -0.4 & Ary \\
\hline
\end{tabular}

Table 8 ADS 5958: O-C residuals of our new orbit (after 2006). The symbol ${ }^{P}$ indicates PISCO measurements.

\begin{tabular}{crrl}
\hline Epoch & $\begin{array}{r}\Delta \rho(\mathrm{O}-\mathrm{C}) \\
\left({ }^{\prime \prime}\right)\end{array}$ & $\begin{array}{r}\Delta \theta(\mathrm{O}-\mathrm{C}) \\
\left({ }^{\circ}\right)\end{array}$ & Observer \\
\hline 2006.269 & 0.003 & $0.7^{P}$ & Pru \\
2008.202 & -0.005 & $2.1^{P}$ & Pru \\
2009.265 & -0.000 & -0.7 & Tok \\
2009.267 & -0.007 & $0.3^{P}$ & Sca \\
2014.201 & 0.011 & $0.1^{P}$ & Sca \\
2016.266 & -0.000 & $0.8^{P}$ & Sca \\
2016.268 & 0.001 & $0.7^{P}$ & Sca \\
2018.133 & 0.005 & $-1.9^{P}$ & Sca \\
\hline
\end{tabular}

$6276,7294,8211$ and 13169 , respectively. The name of the observer is reported in the last column, using the US Naval Observatory convention. The "Epoch" in the first column is the Julian epoch of the observations.

In Table 13, we present some physical parameters of those systems. The (total) visual magnitudes (Col. 3) and the spectral types (Col. 5) were extracted from the SIMBAD data base. The difference of magnitude between the components (Col. 4) was taken from the IC4. The dynamical parallaxes (Col. 6) were derived from our orbital elements using Baize \& Romani (1946)'s method, with our revised formulae presented in Scardia et al. (2008b). In Col. 7, we report the Hipparcos parallaxes from ESA (1997), the revised values from van Leeuwen (2007), or the Gaia values (ESA, 2018) when available, as indicated in Col. 10. In Cols. 8 and 9 , we give the corresponding linear size of the semimajor axis $a$ and the total mass $\mathfrak{M}_{\text {total }}$, respectively, that were computed from our orbital elements and the Hipparcos/Gaia parallaxes.

\section{WDS 01398-5612 - DUN 5 - HIP 7751 ( $p$ Eri)}

This couple was discovered by James Dunlop, in December 1825, with the 4.6-inch $(12 \mathrm{~cm})$ refractor of the Parramatta Observatory in Sydney (Australia). The first measurements were very rough and published in Dunlop (1829). This couple is made of two well separated stars of similar spectral type $\mathrm{K} 2 \mathrm{~V}$ and visual magnitudes 5.78 and 5.90 , which are very close to the sun, at distance of 26.7 light years only $\left(\pi_{\text {Gaia }}=\right.$ 122 mas). Since that epoch, the companion has cov- 

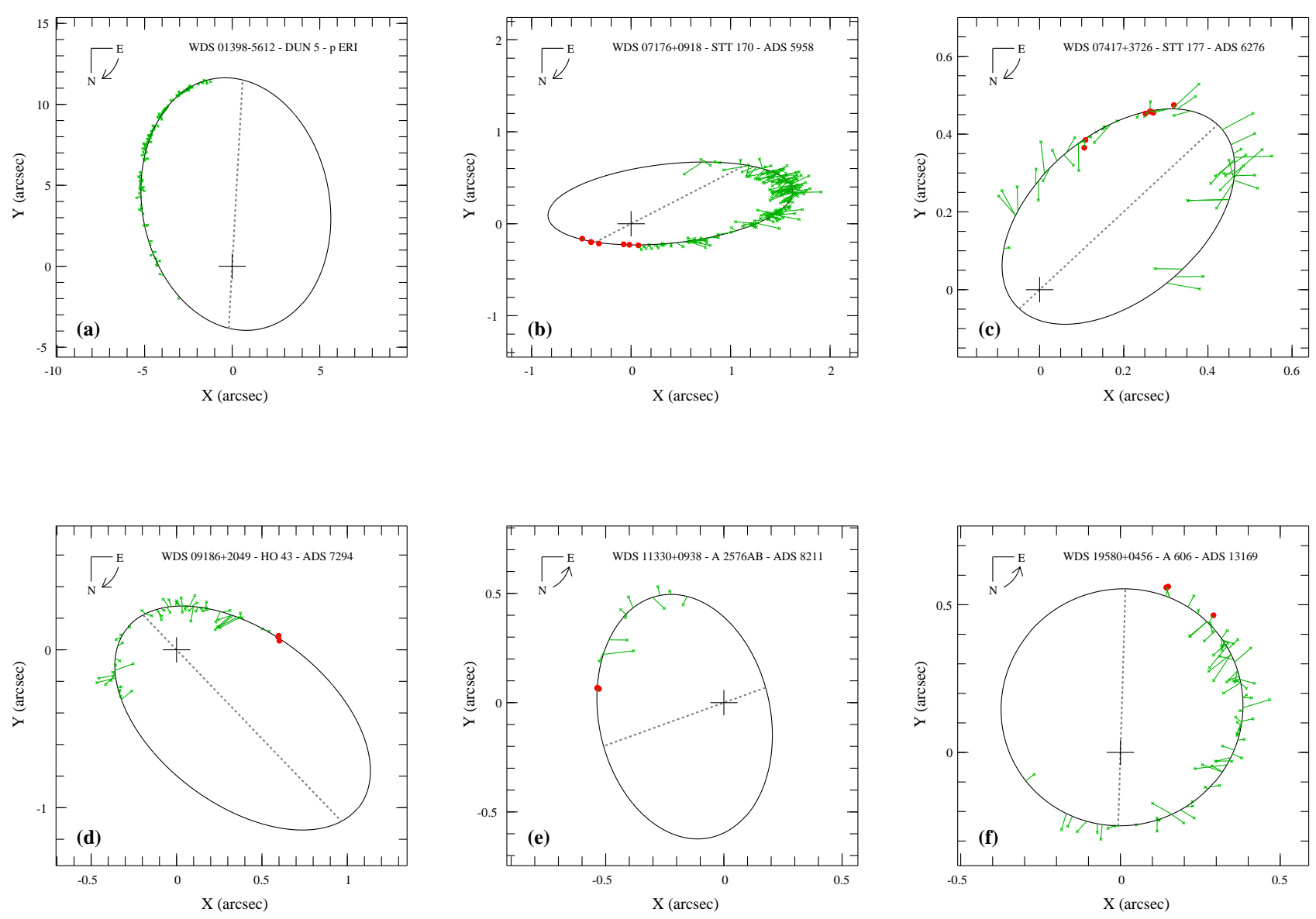

Fig. 8 New orbits of DUN 5 (a), ADS 5958 (b), ADS 6276 (c), ADS 7294 (d), ADS 8211 (e) and ADS 13169 (f). The observations by PISCO are plotted as filled circles that appear in red in the electronic version of this paper.

Table 9 ADS 6276: O-C residuals of our new orbit (after 2007). The symbol ${ }^{P}$ indicates PISCO measurements.

\begin{tabular}{crrl}
\hline Epoch & $\begin{array}{r}\Delta \rho(\mathrm{O}-\mathrm{C}) \\
\left({ }^{\prime \prime}\right)\end{array}$ & $\begin{array}{r}\Delta \theta(\mathrm{O}-\mathrm{C}) \\
\left({ }^{\circ}\right)\end{array}$ & Observer \\
\hline 2007.192 & -0.007 & $0.2^{P}$ & Pru \\
2007.802 & -0.016 & 0.3 & Hrt \\
2008.101 & 0.023 & 1.2 & Gii \\
2009.267 & -0.005 & $0.5^{P}$ & Sca \\
2014.245 & -0.022 & $2.7^{P}$ & Sca \\
2015.272 & -0.025 & $2.5^{P}$ & Sca \\
2018.147 & 0.009 & $0.5^{P}$ & Sca \\
\hline
\end{tabular}

ered an arc of orbit of about $141^{\circ}$, and will reach its apastron in the next few years (see Fig. 8.a). In the last century, good quality measurements were made by using photographic observations with long focal length telescopes located in Southern Africa (Johannesburg) and in Indonesia (Bosscha Observatory, Lembang). After the end of those programs, only a few micrometric measurements have been made by one of us (R. Argyle)
Table 10 ADS 7294: O-C residuals of our new orbit (after 1991). The symbol ${ }^{P}$ indicates PISCO measurements.

\begin{tabular}{crrl}
\hline Epoch & $\Delta \rho(\mathrm{O}-\mathrm{C})$ \\
& $\left(^{\prime \prime}\right)$ & $\Delta \theta(\mathrm{O}-\mathrm{C})$ & Observer \\
$\left({ }^{\circ}\right)$ & \\
\hline 1991.250 & 0.011 & -0.0 & HIP \\
1991.271 & 0.017 & 3.9 & Gii \\
2004.196 & 0.006 & -0.8 & Hrt \\
2008.098 & 0.014 & -0.4 & Gii \\
2016.293 & 0.008 & $1.0^{P}$ & Sca \\
2016.296 & 0.005 & $0.4^{P}$ & Sca \\
2018.346 & -0.004 & $-0.8^{P}$ & Sca \\
\hline
\end{tabular}

with the 67-cm Innes refractor, in Johannesburg, and by R. Anton with CCD imaging and amateur instruments in Namibia. Five orbits have been published up to now, with the last one by Van Albada (1957).

We have taken profit of more than 60 years of additional observations to compute the new orbital elements presented in Table 5, and first published in 
Table 13 Physical parameters $\left(\pi_{\text {dyn }}, a\right.$ and $\left.\mathfrak{M}_{\text {total }}\right)$ derived from the new orbital elements.

\begin{tabular}{|c|c|c|c|c|c|c|c|c|c|}
\hline Name & HIP & $m_{V}$ & $\Delta m_{V}$ & Spectral type & $\begin{array}{l}\pi_{\text {dyn }} \\
(\text { mas })\end{array}$ & $\begin{array}{c}\pi_{H I P / G A I A} \\
(\text { mas })\end{array}$ & $\begin{array}{c}a \\
(\mathrm{AU})\end{array}$ & $\begin{array}{r}\mathfrak{M}_{\text {total }} \\
\left(\mathrm{M}_{\odot}\right) \\
\end{array}$ & Source of $\pi_{\text {HIP } / \text { GAIA }}$ \\
\hline DUN 5 & 7751 & 5.07 & 0.1 & $\mathrm{~K} 2 \mathrm{~V}+\mathrm{K} 2 \mathrm{~V}$ & 110.7 & $\begin{array}{l}122.13 \\
\pm 0.05\end{array}$ & $\begin{aligned} & 65.3 \\
\pm & 0.03^{(1)}\end{aligned}$ & $\begin{aligned} & 1.15 \\
\pm & 0.01^{(1)}\end{aligned}$ & GAIA, ESA (2018) \\
\hline$"$ & $"$ & $"$ & $"$ & $"$ & $"$ & $\begin{array}{l}127.84 \\
\pm 2.19\end{array}$ & $\begin{array}{l}62.4 \\
\pm 1.1^{(1)}\end{array}$ & $\begin{aligned} & 1.00 \\
\pm & 0.05^{(1)}\end{aligned}$ & HIP, van Leeuwen (2007) \\
\hline ADS 5958 & 35310 & 6.77 & 0.3 & G0 V & 24.2 & $\begin{array}{l}22.85 \\
\pm 1.79\end{array}$ & $\begin{array}{r}59.8 \\
\pm 4.8\end{array}$ & $\begin{array}{r}2.6 \\
\pm 0.7\end{array}$ & HIP, ESA (1997) \\
\hline$"$ & $"$ & $"$ & $"$ & $"$ & $"$ & $\begin{array}{l}24.03 \\
\pm 1.04\end{array}$ & $\begin{array}{r}56.8 \\
\pm 2.7\end{array}$ & $\begin{array}{r}2.2 \\
\pm 0.4\end{array}$ & HIP, van Leeuwen (2007) \\
\hline ADS 6276 & 37483 & 7.63 & 1.2 & A3 V & 7.4 & $\begin{array}{l}7.25 \\
\pm 1.4\end{array}$ & $\begin{array}{l}46.1 \\
\pm 8.9^{(1)}\end{array}$ & $\begin{aligned} & 3.2 \\
\pm & 1.8^{(1)}\end{aligned}$ & GAIA, ESA (2018) \\
\hline$"$ & $"$ & $"$ & $"$ & $"$ & $"$ & $\begin{array}{c}5.96 \\
\pm 1.34\end{array}$ & $\begin{aligned} & 56.0 \\
\pm & 12.6^{(1)}\end{aligned}$ & $\begin{aligned} & 5.8 \\
\pm & 3.9^{(1)}\end{aligned}$ & HIP, van Leeuwen (2007) \\
\hline ADS 7294 & 45671 & 8.64 & 0.1 & F5 V & 7.6 & $\begin{array}{l}8.05 \\
\pm 2.45\end{array}$ & $\begin{array}{l}112.4 \\
\pm 34.2^{(1)}\end{array}$ & $\begin{aligned} & 2.1 \\
\pm & 1.9^{(1)}\end{aligned}$ & ) HIP, ESA (1997) \\
\hline$"$ & $"$ & $"$ & $"$ & $"$ & $"$ & $\begin{array}{l}9.25 \\
\pm 1.27\end{array}$ & $\begin{aligned} & 97.8 \\
\pm & 13.4^{(1)}\end{aligned}$ & $\begin{aligned} & 1.4 \\
\pm & 0.6^{(1)}\end{aligned}$ & HIP, van Leeuwen (2007) \\
\hline ADS 8211 & - & 9.47 & 0.1 & G5 V & 10.2 & - & 63.0 & 1.9 & Not available. \\
\hline ADS 13169 & 98272 & 8.70 & 0.1 & $\mathrm{G} 3 \mathrm{~V}+\mathrm{G} 4 \mathrm{~V}$ & 8.4 & $\begin{array}{c}5.63 \\
\pm 0.99\end{array}$ & $\begin{aligned} & 72.8 \\
\pm & 12.8^{(1)}\end{aligned}$ & $\begin{aligned} & 7.9 \\
\pm & 4.2^{(1)}\end{aligned}$ & GAIA, ESA (2018) \\
\hline$"$ & $"$ & $"$ & $"$ & $"$ & $"$ & $\begin{array}{l}7.53 \\
\pm 1.88\end{array}$ & $\begin{aligned} & 54.4 \\
\pm & 13.6^{(1)}\end{aligned}$ & $\begin{aligned} & 3.3 \\
\pm & 2.5^{(1)}\end{aligned}$ & HIP, van Leeuwen (2007) \\
\hline
\end{tabular}

(1) lower estimate of the error, using the parallax error only, and neglecting all the other (unknown) errors.

Table 11 ADS 8211: O-C residuals of our new orbit (after 1980). The symbol ${ }^{P}$ indicates PISCO measurements.

\begin{tabular}{crrl}
\hline Epoch & $\Delta \rho(\mathrm{O}-\mathrm{C})$ \\
& $\left(^{\prime \prime}\right)$ & $\Delta \theta(\mathrm{O}-\mathrm{C})$ & Observer \\
& $\left.{ }^{\circ}\right)$ & \\
\hline 1980.200 & -0.066 & -4.5 & Hei \\
1988.180 & 0.001 & 5.7 & Hei \\
1991.610 & -0.107 & -8.4 & TYC \\
2016.340 & -0.001 & $0.1^{P}$ & Sca \\
2016.342 & -0.002 & $-0.2^{P}$ & Sca \\
2018.346 & -0.008 & $-1.1^{P}$ & Sca \\
\hline
\end{tabular}

the IAU Information Circulars (Scardia et al., 2015b), without any detail of their computation, though. If we except the photographic long focal length measurements, the overall quality of the measurements is poor. In particular, the angular separation measurements are very scattered. Our fit of the 165 available measurements lead to an average rms error of $0^{\prime \prime} .1$ and $0^{\circ} .8$ for $\rho$ and $\theta$, respectively. The sum of the masses of the system that is obtained with Hipparcos or Gaia parallaxes, is of the order of one solar mass (see Table 13), which is slightly smaller than the expected theoretical value of $2 \times 0.79 \mathrm{M}_{\odot}$ for two $\mathrm{K} 2 \mathrm{~V}$ stars according to Straizys \& Kuriliene (1981). The dynamical parallax that is computed with our formula (Scardia, 2008b) is 111 mas, which is slightly smaller than Hipparcos and Gaia's values, and leads to a larger systemic mass of $1.58 M_{\odot}$, which corresponds to the theoretical value.
Table 12 ADS 13169: O-C residuals of our new orbit (after 1998). The symbol ${ }^{P}$ indicates PISCO measurements.

\begin{tabular}{crrl}
\hline Epoch & $\Delta \rho(\mathrm{O}-\mathrm{C})$ \\
$\left({ }^{\prime \prime}\right)$ & $\Delta \theta(\mathrm{O}-\mathrm{C})$ & Observer \\
& 0.031 & $0.1^{P}$ & Sca \\
\hline 1998.679 & -0.068 & 2.6 & Lin \\
1999.504 & -0.068 & 2.3 & Pri \\
1999.504 & 0.013 & 0.7 & WSI \\
2003.784 & 0.042 & -0.3 & Tok \\
2008.767 & 0.032 & $2.0^{P}$ & Sca \\
2016.718 & 0.031 & $1.9^{P}$ & Sca \\
2016.729 & 0.034 & $0.1^{P}$ & Sca \\
2018.375 & & & \\
\hline
\end{tabular}

Our elements are much more accurate than Van Albada (1957)'s elements, but they must still be considered as "provisional", according to the standards commonly used for orbit calculation.

\section{WDS 07176+0918 - STT 170 - ADS 5958 - HIP 35310}

This couple was discovered by O Struve with the 38-cm Merz-Mahler Poulkova refractor, during his survey aimed at founding new double stars (August 1841 - December 1842) (Struve, 1843). The first measurement was made in Dorpat by J.H. Mahler with the famous 25-cm refractor built by Fraunhofer (Mahler, 1844). This couple has been regularly observed since, but the overall quality in angular separation is poor. Six orbits have been published for this couple, including 
our orbit of Scardia et al (2016). Since the companion has moved recently through the periastron, we decided to take profit of the last PISCO observations made after the periastron passage to improve this orbit. Our fit of the 241 available measurements lead to average residuals of $0^{\prime \prime} .101$ and $1^{\circ} .47$ for $\rho$ and $\theta$, respectively. As shown in Table 13, the dynamical parallax $\pi_{\text {dyn }}$ obtained with our formula (Scardia, 2008b) is close to the Hipparcos values and the systemic mass $\mathfrak{M}_{\text {total }}$ is in good agreement with the theoretical expected value of $2.1 \mathrm{M}_{\odot}$ of Straizys \& Kuriliene (1981) .

\section{WDS 07417+3726 - STT 177 - ADS 6276 - HIP 37483}

This couple was also discovered by O Struve with the 38-cm Merz-Mahler Poulkova refractor, during his 1841-1842 survey (Struve, 1843). The first measurement was made in Dorpat by J.H. Mahler with the 25-cm Fraunhofer refractor (Mahler, 1844). This couple has been regularly observed since, but the quality of the measurements made before 1950 is poor. Four orbits have been published until now. The last one, computed by Heintz (1982), begins to show systematic errors on the position angle residuals.

We have computed a new orbit for this couple that was first published in the IAU Information Circulars (Scardia et al., 2016). The recent observation made with PISCO with the 1-m Epsilon telescope of OCA (Obs. de la Côte d'Azur, France) confirm our elements. Our fit of the 54 available measurements lead to average residuals of $0^{\prime \prime} .05$ and $2^{\circ} .70$ for $\rho$ and $\theta$, respectively.

The Hipparcos parallax is 5.96 mas according to Van Leeuwen (2007), and the Gaia value is 7.25 mas (ESA, 2018). Those two values lead to a systemic mass $\mathfrak{M}_{\text {total }}$ of $5.8 M_{\odot}$ and $3.2 M_{\odot}$, respectively (see Table 13). The latter value is in a fair agreement with the theory $\left(4.0 \mathrm{M}_{\odot}\right.$ according to Straizys \& Kuriliene, 1981). The dynamical parallax $\pi_{\text {dyn }}$ obtained with our formula (Scardia, 2008b) is in very good agreement with the Gaia parallax measurement (see Table 13).

\section{WDS 09186+2049 - HO 43 - ADS 7294 - HIP 45671}

This couple was discovered in April 1862 by G.W. Hough with the 47-cm refractor of Dearborn Observatory (Illinois, USA). It is one of the first binaries that have been discovered by this famous observer. In the original publication of his first discoveries (Hough, 1887), this object was reported as "A close and difficult pair". This double star was then regularly observed but the measurements are rather scattered, both for the angular separation and for the position angle, especially at the beginning of the XXth century. The companion has covered an arc of about $210^{\circ}$ in the orbit, and the periastron passage occurred around 1945 . Five orbits have been yet published, and the last one by Baize (1989) does not account any longer for the observations: its period is much too short. We computed a revised version of this orbit using our usual procedure (see above), and published it in the IAU Circular Information (Scardia et al., 2016), without any detail of that computation. Our fit of the 58 measurements lead to mean residuals of $0^{\prime \prime} .05$ and $3^{\circ} .1$ for $\rho$ and $\theta$, respectively. The recent observations made with PISCO at Calern have confirmed this orbit (see Table 10). For estimating the total mass and the semi-major axis in $\mathrm{AU}$, we have only used the Hipparcos parallax measurements since the Gaia-DR2 does not include a value for the parallax. The mass value obtained with the Hipparcos parallax from ESA (1997) is $2.1 \mathrm{M}_{\odot}$, is closer to the theoretical value than what is obtained with van Leeuwen (2007). The dynamical parallax computed with our formula (Scardia et al., 2008b) is 7.6 mas. It is close to the ESA (1997) value and leads to a total "dynamical" mass of $2.63 \mathrm{M}_{\odot}$, also in good agreement with the theoretical value of $2.4 \mathrm{M}_{\odot}$ that is expected for a F5 V stellar system (Straizys \& Kuriliene, 1981).

\section{WDS 11330+0938 - A 2576 AB - ADS 8211}

This couple was discovered by R.G Aitken with the 91-cm refractor of Lick Observatory in May 1913 (Aitken, 1913). It belongs to a wide stellar system of 4 stars that are likely to be gravitationally linked. The CD couple of that system is made of two faint stars $\left(m_{V}=12.0\right.$ and $\left.m_{V}=13.0\right)$ which is reported as J 428 CD in the WDS. The couple A2576 AB has been poorly observed since its discovery and forgotten for many years. Only more than a dozen of observations have been published as a whole, and no orbit has been computed yet. We observed A2576 AB with PISCO and the Epsilon telescope at Calern in 2016 after a gap in the observations of more than 25 years. Until now, the companion has been monitored over an arc of orbit of about $60^{\circ}$, and has been moving towards the apastron (see Fig. 8.f). The available data set is therefore sufficient to determine the first preliminary orbit for A 2576 AB. In 2016, we computed an orbit that was published in the IAU Information Circular (Scardia et al., 2016). The elements have been obtained with the method of Kowalsky (1870), which is based on the selection of the best orbital elements that generate an apparent ellipse that well mach the plot of the actual measurements. The fit of the 13 available measurements lead to mean residuals of $0^{\prime \prime} .04$ and $3^{\circ} .3$ for $\rho$ and $\theta$, respectively. We have not found any trigonometric parallax in the literature for this object, neither from Hipparcos, Gaia nor in the. Yale Trigonometric Parallaxes Catalog (Van Altena et al., 1995). The dynamical parallax of 10.2 mas reported in Table 13 was obtained using our formula of (Scardia et al, 2008b), with a spectral type of G5 V, a total visual magnitude of 9.47 , and a difference of magnitudes of 0.08 . The total dynamical mass would then be $1.85 \mathrm{M}_{\odot}$ which is in fair agreement with the expected value of $2.1 \mathrm{M}_{\odot}$ by the theory (Straizys \& Kuriliene, 1981). 


\section{WDS 19580+0456 - A 606 AB - ADS 13169 - HIP 98272}

This couple was also discovered by R.G. Aitken with the 91-cm refractor of Lick Observatory, in June 1903 (Aitken, 1904). It has been then regularly monitored by the observers. More than five orbits have been published yet. The last one, by Zaera (1982) is now outdated, and does not account for the observations any longer. We observed this object with PISCO and the Epsilon telescope of Calern in Sept. 2016 and computed a new orbit that was published in the IAU Information Circular (Scardia et al., 2016). That observation clearly showed that the companion was in the 2nd quadrant (i.e., South-East), whereas most observations reported in the IC4 are generally in the 4th quadrant (i.e. NorthWest). The few exceptions with a location in the 2nd quadrant are the Hipparcos observation of 1991 and our observation made with PISCO in 1998 (Scardia et al., 2000). The origin of this problem may be due to the small brightness difference of the two components, and a possible switch of the two components between the blue and red part of the spectrum (our observations were made with a red filter, whereas the other observers used a blue or green filter). For our orbit, we took the quadrant convention of the Hipparcos observation, and swapped all the other measurements, when needed. The new orbital elements that we obtained from the 71 available measurements are given in Table 5. They lead to mean residuals of $0^{\prime \prime} .04$ and $2^{\circ} .5$ for $\rho$ and $\theta$, respectively. The total mass inferred from those elements with the Gaia preliminary parallax of 5.6 mas $(\mathrm{ESA}, 2018)$ is $7.9 \mathrm{M}_{\odot}$, which is in excess for a G3V+G4V couple whereas the Hipparcos value of 7.5 mas leads to $3.3 \mathrm{M}_{\odot}$, which is closer to the theoretical value of $2.1 \mathrm{M}_{\odot}$ (Straizys \& Kuriliene, 1981). The dynamic parallax obtained with our formula (Scardia et al., 2008), is 8.4 mas and the dynamical mass is $2.5 \mathrm{M}_{\odot}$, which is fair agreement with the theory. Note that this orbit must be considered as "provisional", and the elements are expected to change in the future.

\section{Conclusion}

We have presented here the 196 new measurements of 173 visual binaries that we have obtained with PISCO in 2015. The average accuracy was $0^{\prime \prime} .019$ for the angular separation and $0^{\circ} .6$ for the position angles.

We also presented new orbital elements computed for DUN 5, ADS 5958, 6276, 7294, 8211, and 13169, that were partly derived from PISCO observations. The total mass values we have obtained are compatible with the expected theoretical values. The dynamical parallaxes computed with our revised formulae presented in Scardia et al. (2008b) are in very good agreement with the Hipparcos and Gaia values, when available. We also computed new rectilinear elements for ADS 4841 for which the physical connection is doubtful from our (and other recent) observations.

The total number of measurements made with PISCO in Merate since its installation in 2003 now exceeds 3700 . Our group has thus provided a good contribution to the continuing monitoring of long period visual binary systems, which is important for refining systemic stellar masses. This paper is the last of this series, since the observations with PISCO in Merate have stopped in June 2015. This instrument was moved to the Epsilon telescope of OCA in Calern (France) in July 2015, and since then we have been continuing our binary star program from there.

Acknowledgements. We are grateful to Giorgio Pariani and Martino Quintavalla from the GOLEM group in Merate for their invaluable help for solving mechanical problems with the dome of the Zeiss telescope. We thank the members of the U.S. Naval Observatory, Washington DC, for kindly sending us some lists of measurements of visual binaries. This work has made use of the Washington Double Star Catalog (http://ad.usno.navy.mil/wds/wds), the "Fourth Catalog of Interferometric Measurements of Binary Stars" (http://ad.usno.navy.mil/wds/int4), and the "Sixth Catalog of Orbits of Visual Binary Stars" (http://ad.usno.navy.mil/wds/orb6), maintained at the U.S. Naval Observatory. We also used the SIMBAD astronomical data base (http://simbad.u-strasbg.fr/simbad) operated by the Centre de Données Astronomiques de Strasbourg (France). This work has also made use of data from the European Space Agency (ESA) mission Gaia (https://www.cosmos.esa.int/gaia), processed by the Gaia Data Processing and Analysis Consortium (DPAC, https://www.cosmos.esa.int/web/gaia/dpac/consortium). Finally, we thank A. Tokovinin, the referee of this paper, for his constructive remarks and suggestions.

\section{References}

Aitken, R.G., 1904, Lick Obs. Bull. 2, 139

Aitken, R.G., 1913, Lick Obs. Bull. 7, 186

Aitken, R.G., 1932, "New General Catalog of Double Stars", Carnegie Institute, Washington

Aristidi, E., Carbillet, M., Lyon, J.-F., Aime, C., 1997, A\&AS, 125, 139

Baize, P., 1980, A\&A Suppl. 39, 83

Baize, P., 1989, A\&A Suppl. 78, 125

Baize, P., Romani, L., 1946, Ann. Astrophys. 9, 13

Dunlop, J.,1829, Mem.R.A.S. 3, 257

ESA: 1997, The Hipparcos and Tycho Catalogues, ESA SP1200, ESA Publications Division, Noordwijk

ESA: 2018, Gaia Archive 2nd release, http::gea.esac.esa.int/archive/

Hartkopf, W.I., Mason, B.D., 2018, "Sixth Catalog of Orbits of Visual Binary Stars" http://ad.usno.navy.mil/wds/orb6.html (OC6)

Hartkopf, W.I., Mason, B.D., Wycoff, G.L., McAlister, H.A., 2018, "Fourth Catalog of Interferometric Measurements of Binary Stars" http://ad.usno.navy.mil/wds/int4.html (IC4)

Heintz, W.D., 1982, A\&A Suppl. 47, 569 
Hellerich, J., 1925, Astron. Nach., 223, 335

Hough, G.W., 1887, Astron. Nachr. 116,273

Kowalsky, M., 1873, Procès-verbaux de l'Université Impériale de Kasan

Mahler, J.H., 1844, Dorpat Observations 11, 3

Mason, B.D., Wycoff, G.L., Hartkopf, W.I., 2018, "Washington Double Star Catalog" http://ad.usno.navy.mil/wds/wds.html (WDS)

Prieur, J.-L, Koechlin, L., André, C., Gallou, G., Lucuix, C., 1998, Experimental Astronomy, vol 8, Issue 4, 297

Prieur, J.-L., Scardia, M., Pansecchi, L., Argyle, R.W., Sala, M., Ghigo, M., Koechlin, L., Aristidi, E., 2008, MNRAS, 387, 772 (Paper V)

Prieur, J.-L., Scardia, M., Pansecchi, L., Argyle, R.W., Sala, M., 2009, MNRAS, 395, 907 (Paper VII)

Prieur, J.-L., Scardia, M., Pansecchi, L., Argyle, R.W., Sala, M., 2010, MNRAS, 407, 1913 (Paper IX)

Prieur, J.-L., Scardia, M., Pansecchi, L., Argyle, R.W., Sala, M., 2012, MNRAS, 422, 1057 (Paper XI)

Prieur, J.-L., Scardia, M., Pansecchi, L., Argyle, R.W., Zanutta, A., Aristidi, E., 2014, Astron. Nach., 335, 817 (Paper XIII)

Prieur, J.-L., Scardia, M., Pansecchi, L., Argyle, R.W., Zanutta, A., Aristidi, E., 2017, Astron. Nach., 338, 74 (Paper XV)

Scardia, M., Prieur, J.-L., Aristidi, E., Koechlin L., 2000, ApJ Suppl., 131, 561

Scardia, M., Prieur, J.-L., Sala, M., Ghigo, M., Koechlin, L., Aristidi, E., Mazzoleni, F., 2005, MNRAS, 357, 1255 (with erratum in MNRAS 362, 1120) (Paper I)

Scardia, M., Prieur, J.-L., Pansecchi, L., Argyle, R.W., Sala, M., Ghigo, M., Koechlin, L., Aristidi, E., 2006, MNRAS, 367, 1170 (Paper II)

Scardia, M., Prieur, J.-L., Pansecchi, L., Argyle, R.W., Basso, S., Sala, M., Ghigo, M., Koechlin, L., Aristidi, E., 2007, MNRAS, 374, 965 (Paper III)

Scardia, M., Prieur, J.-L., Pansecchi, L., Argyle, R.W., Sala, M., Basso, S., Ghigo, M., Koechlin, L., Aristidi, E., 2008a, Astron. Nach., 329, 1, 54 (Paper IV)

Scardia, M., Prieur, J.-L., Pansecchi, L., Argyle, R.W., 2008b, Astron. Nach., 329, 379

Scardia M., Prieur J.-L., Pansecchi L., Argyle R.W., Sala M., 2009, Astron. Nach., 330, 1, 55 (Paper VI)

Scardia, M., Prieur, J.-L., Pansecchi, L., Argyle, R.W., Sala, M., 2010, Astron. Nach., 331, 286 (Paper VIII)

Scardia, M., Prieur, J.-L., Pansecchi, L., Argyle, R.W., Sala, M., 2011, Astron. Nach., 332, 508 (Paper X)

Scardia, M., Prieur, J.-L., Pansecchi, L., Argyle, R.W., Spanó, P., Riva, M., Landoni, M., 2013, MNRAS, 434, 2803 (Paper XII)

Scardia, M., Prieur, J.L., Pansecchi, L., Argyle, R.W., Zanutta, A., Aristidi, E., 2015a, Astron. Nachr., 336, 388 (Paper XIV)

Scardia, M. et al, 2015b, IAU Commission G1, Information Circular n. 186

Scardia, M. et al, 2016, IAU Commission G1, Information Circular n. 189

Straizys, V., Kuriliene, G., 1981, AP\&SS, 80, 353.

Struve, O., 1843, "Catalogue de 514 étoiles doubles et multiples", Académie Impériale des Sciences, St. Petersbourg van Albada, G.B., 1957, Contr. Bosscha Obs. n. 5

van Altena, W.F., Lee, J.T., Hoffleit, E.D., 1995, "The general catalogue of trigonometric stellar parallaxes", New
Haven, CT: Yale University Observatory

van Leeuwen, F., 2007, "Hipparcos, the new reduction of the raw data", Springer Netherlands Ed.

Zaera, J.C., 1982, IAU Commission n. 26, Information Circular n. 87 